Samuel Amorim de Souza

\title{
EFEITO DE UMA SESSÃO AGUDA DE EXERCÍCIO RESISTIDO COM MODERADA RESTRIÇÃO DO FLUXO SANGUÍNEO NA SAÚDE VASCULAR E COMPORTAMENTO HEMODINÂMICO EM IDOSOS COM BAIXA VELOCIDADE DE MARCHA
}

Dissertação apresentada à Faculdade
Israelita de Ciências da Saúde Albert
Einstein para obtenção do Título de Mestre
em Ciências da Saúde.


Samuel Amorim de Souza

\title{
EFEITO DE UMA SESSÃO AGUDA DE EXERCÍCIO RESISTIDO COM MODERADA RESTRIÇÃO DO FLUXO SANGUÍNEO NA SAÚDE VASCULAR E COMPORTAMENTO HEMODINÂMICO EM IDOSOS COM BAIXA VELOCIDADE DE MARCHA
}

\author{
Dissertação apresentada à Faculdade \\ Israelita de Ciências da Saúde Albert \\ Einstein para obtenção do Título de Mestre \\ em Ciências da Saúde. \\ Orientadora: Profa. Dra. Luciana Diniz \\ Nagem Janot de Matos
}




\section{S729e}

Souza, Samuel Amorim de

Efeito de uma sessão aguda de exercício resistido com moderada restrição do fluxo sanguíneo na saúde vascular e comportamento hemodinâmico em idosos com baixa velocidade de marcha / Samuel Amorim de Souza. -- São Paulo, 2020.

$\mathrm{xi} ; 38 \mathrm{f}$.

Dissertação (Mestrado) - Faculdade Israelita de Ciências da Saúde Albert Einstein. Instituto Israelita de Ensino e Pesquisa Albert Einstein. Programa de Pós-Graduação em Ciências da Saúde.

Título em inglês: Effects of acute resistance exercise with moderate blood flow restriction on arterial stiffness and hemodynamic behaviour in elderly people with low gait speed.

1. KAATSU (não DeCS). 2. Rigidez vascular. 3. Fluxo sanguíneo regional. 4. Idoso. 5. Marcha.

NLM - QT 256

Elaborada pelo Sistema Einstein Integrado de Bibliotecas 
FACULDADE ISRAELITA DE CIÊNCIAS DA SAÚDE ALBERT EINSTEIN

Coordenador do Curso de Pós-Graduação: Prof. Dr. Luiz Vicente Rizzo 
Samuel Amorim de Souza

\title{
EFEITO DE UMA SESSÃO AGUDA DE EXERCÍCIO RESISTIDO COM MODERADA RESTRIÇÃO DO FLUXO SANGUÍNEO NA SAÚDE VASCULAR E COMPORTAMENTO HEMODINÂMICO EM IDOSOS COM BAIXA VELOCIDADE DE MARCHA
}

Presidente da banca: Profa. Dra. Luciana Diniz Nagem Janot de Matos

\author{
BANCA EXAMINADORA
}

Membros titulares:

Prof. Dr. Eduardo Juan Troster

Prof. Dr. Gilberto Candido Laurentino

Prof. Dr. Allan Robson Kluser Sales

Membros suplentes:

Profa. Dra. Luciana Cavalheiro Marti

Dr. Hélcio Kanegusuku

Aprovada em: 10/07/2020. 


\section{Dedicatória}

Dedico esse trabalho a minha família, especialmente minha mãe, Maria da Conceição, e ao meu pai, José Orlando.

A minha avó, Elza Lara, que foi minha primeira aluna após certificação como especialista KAATSU e grande inspiração para mudança profissional e despertar para o propósito da minha vida. 


\section{Agradecimentos}

Primeiramente gostaria de agradecer minha família, pelo incentivo e amparo nessa nova jornada pessoal e profissional.

A todos nossos pacientes, pelo compromisso e vital contribuição para realização do nosso estudo.

A equipe de secretárias, enfermeiras, fisioterapeutas e profissionais de educação física do ambulatório Albert Einstein - Vila Mariana que deram todo apoio e suporte.

A Sandra Malagutti, estatística responsável pela análise estatística dos dados.

Aos pesquisadores Paulo Longano e Camila Jordão que ajudaram na coleta de dados.

A Profa. Dra. Alexandra Gaspar, pelo aprendizado e motivação nos momentos difíceis. Considero-te fundamental para minha formação clínica e acadêmica.

Ao Prof. Hans Degens, pela parceria e dedicação nas revisões dos artigos publicados ao longo do mestrado.

A minha orientadora, Profa. Dra. Luciana Janot, pela paciência, confiança e oportunidade de realizar esse projeto de pesquisa. Sempre atenciosa e dedicada em todos os momentos. Eternamente grato pelos ensinamentos!

A Prouniemp, pela bolsa de estudo recebida durante a realização do projeto de pesquisa.

Por fim, agradeço à instituição FAPESP pelo apoio financeiro para realização deste estudo (Processo: 2016/07993-3). 


\section{Sumário}

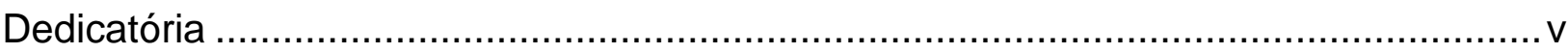

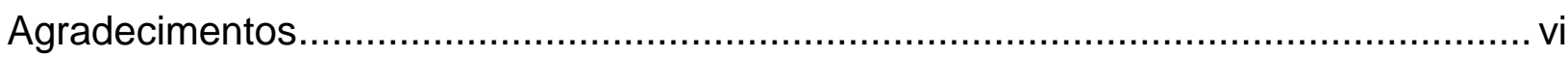

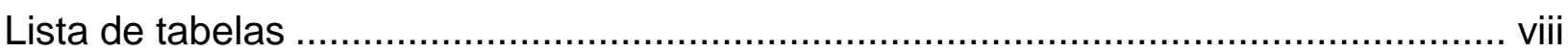

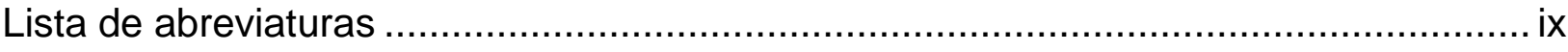

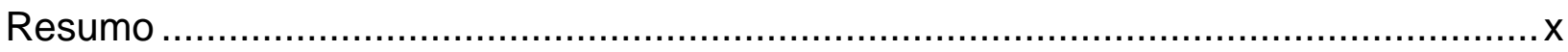

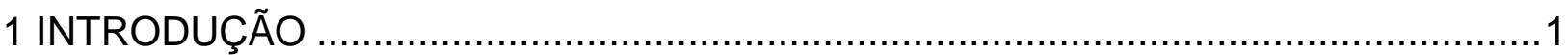

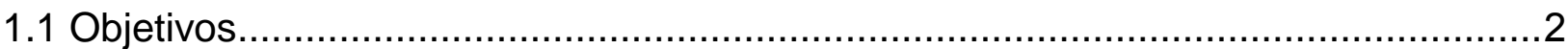

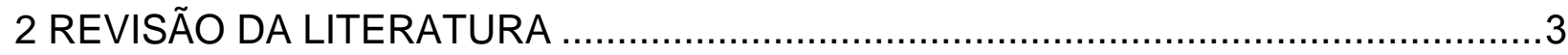

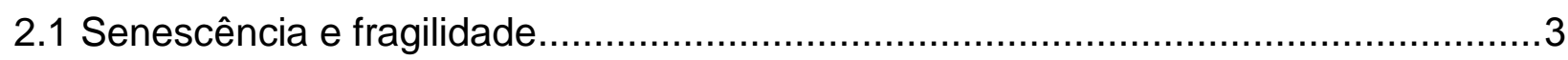

2.2 Senescência e sistema musculoesquelético ................................................

2.3 Senescência, sistema cardiovascular e resposta aguda ao exercício .....................5

2.4 Exercício com restrição do fluxo sanguíneo ...........................................................

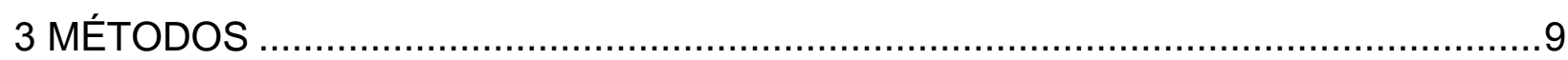

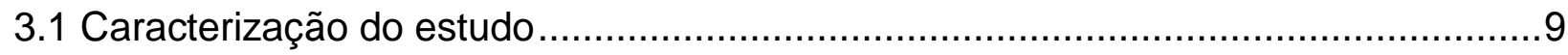

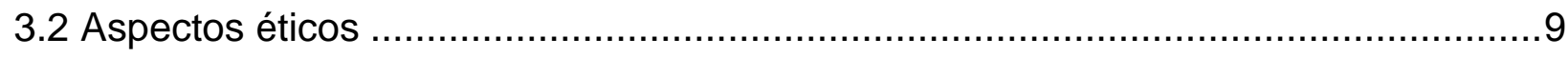

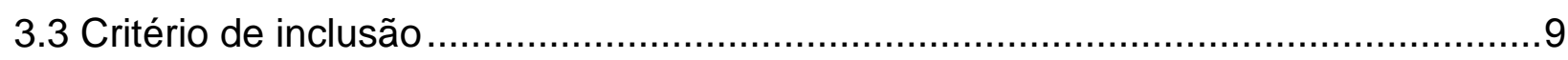

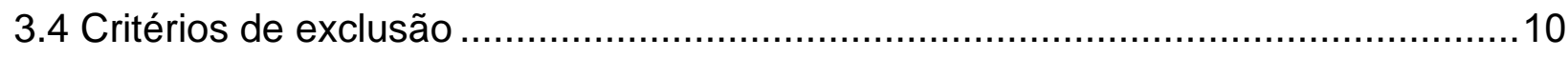

3.5 Protocolo agudo: sessão de exercício resistido.......................................... 10

3.5.1 Teste de 1RM na cadeira extensora e leg press sentado.................................12

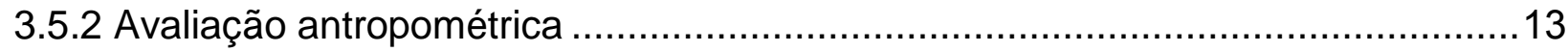

3.5.3 Determinação da pressão da restrição do fluxo sanguíneo .............................13

3.5.4 Avaliação do fluxo sanguíneo muscular ................................................. 14

3.5.5 Medida da rigidez arterial - pressão arterial central e velocidade da onda de pulso

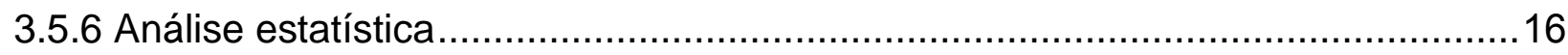

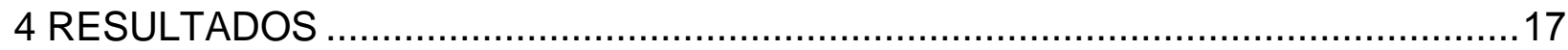

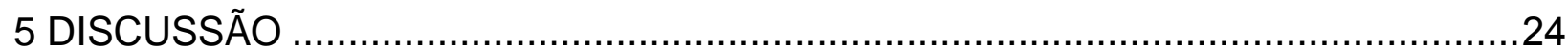

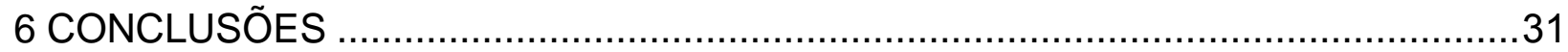

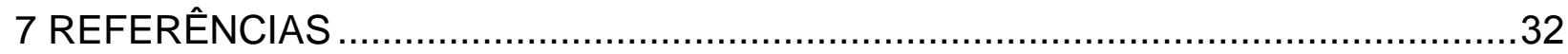

Abstract 


\section{Lista de tabelas}

Tabela 1. Protocolo da sessão aguda de exercício resistido .................................. 12

Tabela 2. Características físicas e dados basais dos participantes .......................... 17

Tabela 3. Comorbidades e doenças associadas aos participantes do estudo .............18

Tabela 4. Medicações dos participantes ..................................................... 19

Tabela 5. Indicadores da pressão arterial central e velocidade da onda de pulso ........20

Tabela 6. Medidas em repouso obtidas na pletismografia de oclusão venosa .............21

Tabela 7. Picos das medidas durante o handgrip obtidas pela pletismografia de oclusão

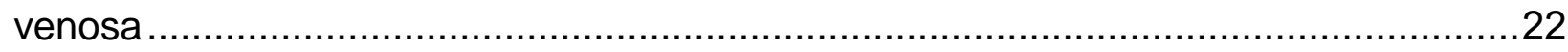

Tabela 8. Variáveis hemodinâmicas pré e pós-sessão aguda de exercício resistido....23 


\section{Lista de abreviaturas}

1RM 1 repetição máxima

Alx Índice de aumentação da pressão aórtica

Alx75 Índice de aumentação da pressão aórtica corrigido a 75bpm

CVM Contração voluntária máxima

ERRF Exercício resistido com moderada restrição do fluxo sanguíneo

ERT Exercício resistido tradicional

FC Frequência cardíaca

PA Pressão arterial

PAC Pressão arterial central

PAD Pressão arterial diastólica

PAM Pressão arterial média

PAS Pressão arterial sistólica

PDA Pressão de aumentação

POV Plestismografia de oclusão venosa

PP Pressão de pulso

RAC Regulação autonômica cardiovascular

TA Tonometria de aplanação

VOP Velocidade da onda de pulso 


\section{Resumo}

Introdução: $O$ exercício resistido agudo de alta intensidade provoca alterações no sistema vascular e no comportamento hemodinâmico, não sendo interessante para população idosa e com comorbidades. Novas estratégias de treinamento, com o uso de exercícios com baixa intensidade associados a moderada restrição do fluxo sanguíneo tem surgido, como o KAATSU. Entretanto, a ação aguda sobre a saúde vascular e comportamento hemodinâmico em idosos com comorbidades e diminuída performance física não é conhecida. Objetivos: Verificar os efeitos de uma sessão aguda do exercício resistido com moderada restrição do fluxo sanguíneo na pressão arterial central, rigidez arterial, fluxo sanguíneo muscular em idosos com baixa velocidade de marcha e verificar o comportamento da pressão arterial e frequência cardíaca até 1 hora após a sessão. Métodos: Estudo randomizado e controlado, composto por dois grupos: exercício resistido tradicional $(60 \% 1 \mathrm{RM})$ e exercício com moderada restrição do fluxo sanguíneo e baixa intensidade (20\% 1RM). Antes e após $1 \mathrm{~h}$ sessão de exercício, a pressão arterial central, rigidez arterial, fluxo sanguíneo muscular, pressão arterial, e frequência cardíaca foram mensuradas. Os grupos foram comparados quanto às medidas iniciais por testes exatos de Fisher para as variáveis qualitativas e teste $t$ de Student no caso das variáveis quantitativas. Para as medidas observadas em mais de um momento, utilizamos modelos de equação de estimação generalizada. $O$ valor-p $<0,05$ foi adotado como estatisticamente significante e todos os dados serão apresentados como média e desvio padrão. Resultados: A amostra do estudo foi composta por 17 idosos ( 3 homens e 14 mulheres), sendo 7 no grupo ERT e 10 no grupo ERRF. O exercício resistido com moderada restrição do fluxo sanguíneo provocou aumento significativo na pressão de aumentação $(p=0,026)$, índice de aumentação da pressão aórtica $(p=0,008)$, índice de aumentação da pressão aórtica corrigido a $75 \mathrm{bpm}(\mathrm{p}=0,014)$ e pressão diastólica aórtica $(p=0,029)$ e radial $(p=0,017)$ em relação ao exercício resistido tradicional. Entretanto, não houve diferença significativa entre os grupos em relação a velocidade de onda de pulso, fluxo sanguíneo muscular e parâmetros hemodinâmicos avaliados até 1 hora após a sessão de exercício realizada. Conclusão: Uma sessão aguda de exercício resistido com moderada restrição do fluxo sanguíneo promove aumento nos parâmetros indiretos de rigidez arterial, pressão diastólica radial e aórtica, pressão de aumentação, índice de 
aumentação da pressão aórtica e índice de aumentação da pressão aórtica corrigido a 75bpm, entretanto não provoca modificações na velocidade da onda de pulso, fluxo sanguíneo muscular e parâmetros hemodinâmicos em idosos com baixa velocidade de marcha.

Identificador ClinicalTrials.gov: NCT 03272737

Descritores: KAATSU (não DeCS); Rigidez vascular; Fluxo sanguíneo regional; Marcha, Idoso 


\section{INTRODUÇÃO}

De acordo com a Organização Mundial da Saúde, em países como China, Tailândia e Brasil ocorrerá um aumento do percentual populacional de pessoas acima de 65 anos de $7 \%$ no ano 2000 para $14 \%$ em 2030. ${ }^{(1)}$

Esses números representam um grande desafio na área da saúde, pois a senescência, compreendida por um processo natural de envelhecimento, está associada a diversas síndromes e fenômenos moleculares e celulares que modificam o sistema cardiovascular, músculo esquelético, com impacto direto na capacidade funcional dos idosos. ${ }^{(2,3)}$

A redução da capacidade funcional está relacionada com a diminuição da velocidade de caminhar, indicador físico associado a síndrome da fragilidade na população idosa. ${ }^{(4,5)}$ Importante salientar, que a velocidade de marcha está associada à sobrevida em idosos. Acrescentar $0,1 \mathrm{~m} / \mathrm{s}$ na velocidade de marcha é capaz de elevar a taxa de sobrevida nessa população. ${ }^{(6)}$

A síndrome da fragilidade é um preditor de mortalidade e desfechos clínicos não favoráveis em idosos. ${ }^{(7)}$ Por isso, a busca por novas estratégias que possam prevenir e minimizar os efeitos do envelhecimento torna-se de extrema importância, principalmente para manutenção da mobilidade e das atividades cotidianas que são fundamentais para a independência do idoso. ${ }^{(8)}$

O treinamento resistido com intensidades até $80 \%$ de uma repetição máxima (1RM) tem sido utilizado e indicado para minimizar perda de força e massa muscular nessa população. ${ }^{(9)}$ No entanto, cargas elevadas podem provocar alterações hemodinâmicas e vasculares, sendo prejudiciais, principalmente em idosos frágeis e com comorbidades associadas. ${ }^{(10)}$

Para tentar diminuir esses efeitos adversos, o exercício resistido com restrição do fluxo sanguíneo pode ser uma ferramenta útil e eficaz para melhora da força e massa muscular em idosos. ${ }^{(11)}$ Entretanto, estudos com foco na rigidez arterial e na vasodilatação muscular em idosos frágeis ainda são limitados, principalmente em populações com maior comprometimento funcional e comorbidades associadas como hipertensão arterial sistêmica, diabetes e obesidade. ${ }^{(12)}$

Portanto, a realização de um estudo randomizado, controlado para analisar a segurança e o efeito de uma sessão aguda do exercício resistido com 
restrição de fluxo sanguíneo no sistema vascular e comportamento hemodinâmico em relação ao exercício resistido tradicional se torna fundamental, para esclarecer dados ainda não suficientemente explorados na literatura e permitir que essa modalidade de exercício possa ser aplicada, em caso de resultados favoráveis.

\subsection{Objetivos}

1. Comparar o efeito agudo entre uma sessão de exercício resistido tradicional com o exercício resistido com moderada restrição do fluxo sanguíneo na pressão arterial central e rigidez arterial em idosos com baixa velocidade de marcha;

2. Comparar o efeito agudo entre uma sessão de exercício resistido tradicional com o exercício resistido com moderada restrição do fluxo sanguíneo no fluxo sanguíneo muscular e condutância vascular em idosos com baixa velocidade de marcha;

3. Comparar o efeito entre uma sessão de exercício resistido tradicional com o exercício resistido com moderada restrição do fluxo sanguíneo no comportamento da pressão arterial e frequência cardíaca até 60 minutos após o exercício. 


\section{REVISÃO DA LITERATURA}

\subsection{Senescência e fragilidade}

A definição clínica de fragilidade se caracteriza por baixa velocidade de marcha, queda da força de preensão palmar, redução das atividades físicas, exaustão, perda de peso não intencional e diminuição das relações sociais do idoso. Quando o idoso apresenta um ou dois critérios pode ser caracterizado pré-frágil. Incluindo três ou mais critérios, é considerado frágil. ${ }^{(13)}$ No Brasil, existe uma prevalência de fragilidade em indivíduos acima de 65 anos que varia entre 10 e $25 \%$. Em idosos com idade superior a 85 anos, esse percentual chega a $46 \% .^{(14)}$

Disfunções no equilíbrio e na marcha são comuns em idosos frágeis devido a disfunções nos sistemas musculoesquelético, vascular e neurológico. A limitação do movimento é um dos principais fatores da perda de independência do idoso, impactando negativamente suas capacidades funcionais diárias. ${ }^{(15)}$

Associado ao processo de senescência, o acúmulo de comorbidades ao longo da vida tem uma relação direta com doenças cardiovasculares. ${ }^{(16)}$ Esse processo é capaz de desencadear um declínio progressivo do estado funcional e cognitivo dos indivíduos, aumentando exponencialmente o número de idosos com a síndrome da fragilidade e consequente repercussão no grau de dependência, internações e geração de custos ao sistema de saúde. ${ }^{(17,18)}$

Neste contexto, medidas capazes de permitir um maior número de idosos com envelhecimento saudável e de reverter as alterações fisiopatológicas que ocorrem com a síndrome de fragilidade são de fundamental importância. Dentre os recursos disponíveis, o exercício físico tem ganhado grande destaque, tanto como uma medida preventiva, quanto como forma de tratamento. ${ }^{(19)}$

\subsection{Senescência e sistema musculoesquelético}

A senescência celular associada à disfunção do sistema musculoesquelético é causada por diversos fatores, tais como: acúmulo de danos ao 
ácido desoxirribonucleico (DNA), deformação das proteínas e o encurtamento dos telômeros. ${ }^{(20)}$

Diversas alterações no tamanho e na composição do músculo, modificam a ativação das fibras musculares, reduzindo a produção de energia, reparação e regeneração muscular, proporcionando perda da massa muscular e capacidade de gerar força. ${ }^{(21)}$

Consequentemente, há fraqueza muscular, osteopenia e sarcopenia, que estão associados a déficits de equilíbrio e alterações de marcha, predispondo o idoso a quedas e mais limitações funcionais, formando um ciclo vicioso, que muitas vezes desencadeia internação prolongada ou mesmo o óbito. ${ }^{(22)}$

Idosos com fraqueza muscular aumentam em 2,6 vezes os riscos para severas limitações de mobilidade, 4,3 vezes os riscos para baixa velocidade de marcha, 2,1 vezes os riscos para mortalidade quando comparados com idosos com maior força muscular. ${ }^{(23)}$

O declínio da massa muscular com o envelhecimento é observado principalmente nos membros inferiores e está associado há uma perda de 30 a $40 \%$ na fibra muscular entre a segunda e oitava década de vida. ${ }^{(24)}$ No entanto, a redução no tamanho da fibra muscular é específica. As fibras tipo I são menos afetadas com o envelhecimento, ${ }^{(25)}$ enquanto as fibras tipo II apresentam uma diminuição de 10 a 40\% no tecido muscular quando comparados a indivíduos jovens. ${ }^{(26)}$

As mudanças específicas no tipo de fibra muscular podem ser entendidas pelo remodelamento das unidades motoras que ocasionam maior denervação das fibras musculares tipo II. ${ }^{(27)}$ Essas modificações do tipo de fibra rápida (Tipo II) para lenta (Tipo I) estão associadas com o envelhecimento, afetando principalmente as fibras musculares tipo IIx..$^{(21)}$

Como consequência das alterações na estrutura e composição das fibras musculares há perda de força e potência muscular, que também é decorrente da diminuição da capacidade intrínseca das fibras do tecido musculoesquelético em promover força. ${ }^{(27)}$ 


\subsection{Senescência, sistema cardiovascular e resposta aguda ao exercício}

O envelhecimento é um dos fatores de risco mais importantes que afeta diretamente a homeostase cardiovascular. ${ }^{(28)} \mathrm{O}$ processo de senescência cardiovascular está associado a diversas alterações nos padrões de expressão dos MicroRNAs, autofagia celular, migração/proliferação das células musculares lisas e calcificação arterial, causando aumento progressivo da rigidez vascular. ${ }^{(28)}$ Além disso, o envelhecimento cardiovascular está associado com processo de aterosclerose, doença arterial coronariana, hipertensão arterial sistêmica, insuficiência cardíaca e fibrilação atrial. ${ }^{(29)}$

Clinicamente, o estudo de Framingham, mostrou que existe uma correlação do envelhecimento com 0 aumento da velocidade da onda de pulso carótida/femoral e da pressão arterial média (PAM). ${ }^{\left({ }^{30}\right)}$ A rigidez arterial provoca modificações hemodinâmicas, causando elevação da pressão sistólica e redução da pressão diastólica, aumentando assim a pressão de pulso (PP) e a velocidade da onda de pulso aórtico. ${ }^{(31)}$

Atualmente, o método padrão ouro para avaliação da rigidez arterial é a tonometria de aplanação (TA) que mensura a velocidade da onda de pulso. Estudo longitudinal reportou que o aumento de $1 \mathrm{~m} / \mathrm{s}$ na velocidade da onda de pulso aórtica eleva em $15 \%$ o risco de eventos cardiovasculares e mortalidade cardiovascular. ${ }^{(32)}$ Além da velocidade de onda de pulso (VOP), por meio da TA também é possível mensurar a rigidez arterial de forma indireta utilizando o índice de aumentação da pressão aórtica (Alx), representado pela diferença entre o segundo e primeiro pico sistólico, dividido pela PP multiplicado por 100. Apesar do Alx ser dependente da rigidez da parede arterial, o efeito do reflexo da onda é determinado pela resistência vascular periférica. O envelhecimento leva a um aumento da VOP pela perda da elasticidade das artérias, o que faz com que a onda refletida chegue de forma mais precoce na raiz da aorta, durante a sístole, ocasionando aumento do Alx. ${ }^{(33)}$ Diversas variáveis podem influenciar a medida do Alx dentre elas a frequência cardíaca, o que torna a medida do Alx normalizado para 75 batimentos por minuto (Alx 75) fundamental. ${ }^{(34)}$

O exercício resistido de alta intensidade pode aumentar o risco e causar aumento da rigidez arterial em idosos. Fisiologicamente, cargas elevadas e 
simultaneamente manobra de Valsava podem aumentar agudamente a pressão arterial (PA) e cronicamente a atividade simpática, contribuindo para o aumento da rigidez arterial. No entanto, o treinamento resistido de baixa intensidade não causa alterações plasmáticas nos níveis de norepinefrina e aumenta os níveis de óxido nítrico no endotélio, melhorando a função vasodilatadora em idosos. ${ }^{(35)}$

No endotélio, ocorrem alterações significativas na expressão, na regulação e atividade do óxido nítrico sintase, proporcionando maior formação de espécies reativas de oxigênio. $O$ processo de envelhecimento contribui para diminuição da capacidade regenerativa do endotélio e da senescência endotelial, sendo caracterizada pelo aumento da taxa de apoptose das células endoteliais.

Uma metodologia centenária, confiável e eficaz utilizada para mensurar a função vasodilatadora em resposta ao exercício físico, especialmente em idosos e pacientes com problemas cardiovasculares é a pletismografia de oclusão venosa (POV). ${ }^{(36-39)}$ Este método permite a avaliação do fluxo sanguíneo muscular e quando associado a medida da PA é possível realizar o cálculo da condutância (fluxo/gradiente de pressão) e resistência vascular (gradiente de pressão/fluxo). Mudanças na condutância determinam um melhor índice de resposta vasomotora local em relação a mudanças na resistência. ${ }^{(40)}$

Estudo em mulheres com hipertensão arterial sistêmica demonstrou que após a realização de exercício resistido de alta intensidade (80\%1RM) há maior elevação do fluxo sanguíneo no antebraço quando comparado ao exercício de moderada intensidade (50\%1RM). ${ }^{(41)}$ Em homens jovens saudáveis, apenas o exercício resistido de alta intensidade ( $70 \% 1 \mathrm{RM}$ ) comparado com baixa intensidade ( $20 \% 1 \mathrm{RM})$ combinado com ou sem restrição do fluxo sanguíneo, causou grande aumento da complacência arterial e na condutância vascular nas pernas. ${ }^{(42)}$ Portanto, diversos estudos já exploraram o método para investigar o endotélio vascular na saúde e na doença. ${ }^{(37)}$

Os parâmetros hemodinâmicos frequência cardíaca $(F C)$ e pressão arterial (PA), normalmente aumentam seus valores após o exercício resistido tradicional (ERT). ${ }^{(43)}$ Além disso, o ERT com intensidade moderada (65\% 1RM) pode promover o aumento desses parâmetros em jovens e idosos. ${ }^{(44)}$ Alguns mecanismos relacionados a regulação autonômica cardiovascular (RAC) são induzidos pelo ERT, tais como: comando central, o reflexo pressor do exercício e o barorreflexo arterial. Durante o ERT, o comando central e o reflexo pressor do exercício são ativados. Após 
isso, sinais neurais controlam o recrutamento de unidades motoras pela contração muscular e estimulam diretamente áreas da RAC no tronco cerebral. Além disso, ocorre ativação de neurônios aferentes do músculo esquelético (mecanorreflexo e metaborreflexo) que compõem o reflexo pressor do exercício. O ERT produz aumento da atividade nervosa simpática e reduz a atividade nervosa parassimpática, modulado pelo barorreflexo arterial. Estes ajustes autonômicos proporcionam o aumento da $\mathrm{FC} \mathrm{e}$ PA. ${ }^{(45)}$

Dessa forma, a utilização desses métodos não invasivos se torna fundamental para verificar os efeitos do exercício resistido agudo no sistema cardiovascular, especialmente na rigidez arterial, vasodilatação muscular e comportamento hemodinâmico em idosos frágeis.

O Colégio Americano de Medicina Esportiva, preconiza intensidades $>65 \%$ 1RM no exercício resistido para ganhos em hipertrofia muscular. ${ }^{(46)} \mathrm{Na}$ prática, aplicar esse treinamento torna-se difícil na população de idosos frágeis e principalmente em pacientes com doenças cardiovasculares devido ao enorme stress gerado no sistema cardiovascular. ${ }^{(35)}$

Apesar de o exercício resistido ser considerado benéfico e seguro para a reabilitação cardíaca, estudos mostram que intensidades elevadas podem gerar prejuízo na rigidez arterial em jovens e indivíduos hipertensos. ${ }^{(47-49)}$

\subsection{Exercício com restrição do fluxo sanguíneo}

$\mathrm{Na}$ busca de exercícios alternativos para ganho de massa muscular e força, o exercício resistido de baixa intensidade com moderada restrição do fluxo sanguíneo (ERRF) tem sido utilizado em jovens, ${ }^{(50,51)}$ atletas, ${ }^{(52)}$ idosos e pacientes com doenças crônicas. ${ }^{(53-59)}$

Estudo com treinamento concorrente (exercício resistido e aeróbico realizados juntos) com cargas de treinamento de $70-80 \%$ de $1 \mathrm{RM}$ comparado com ERRF (20-30\% de 1RM) em idosos sedentários, promoveu resultados similares no aumento da área de secção transversa do quadríceps e do $\mathrm{VO}_{2}$ máxima, ou seja, o ERRF pode ser uma ferramenta importante no tratamento da fragilidade em idosos, favorecendo o aumento da força e massa muscular sem, provavelmente, causar grande impacto no sistema cardiovascular. ${ }^{(60)}$ 
Embora os benefícios do ERRF na função muscular em idosos sejam uma realidade ${ }^{(61-63)}$ são inconsistentes e limitados os estudos que verificaram os efeitos do ERRF na função endotelial, especificamente no fluxo sanguíneo muscular e complacência arterial em idosos. ${ }^{(64,65)}$ Um estudo verificou que o ERRF em indivíduos de meia idade aumentou discretamente a rigidez arterial periférica. ${ }^{(66)}$ Por outro lado, outro estudo realizado com ERRF em idosos saudáveis, aumentou força máxima e área de secção transversa muscular, sem afetar a rigidez arterial. ${ }^{(67)}$

Recentemente, foi demonstrado que o ERRF melhorou a circulação sanguínea periférica e a função endotelial vascular de idosos saudáveis. ${ }^{(68)}$ Além disso, mostrou melhora no fluxo sanguíneo nos membros inferiores em idosos sedentários. ${ }^{(69)}$ No entanto, não foi verificado resultado significativo no fluxo sanguíneo muscular nos membros superiores em idosos saudáveis. ${ }^{(70)}$

Diante dessas controvérsias e da possibilidade do ERRF ser de grande aplicação na população idosa, resultando em aumento de força e massa muscular e consequentemente melhora na funcionalidade, torna-se fundamental verificar o efeito agudo sobre variáveis vasculares e o comportamento hemodinâmico após sua aplicação entendendo a segurança deste método. 


\section{MÉTODOS}

\subsection{Caracterização do estudo}

Trata-se de estudo clínico prospectivo randomizado, controlado, em idosos com baixa velocidade de marcha, acompanhados no ambulatório de geriatria da Unidade Vila Mariana do Hospital Israelita Albert Einstein e em centros parceiros de atendimento de idosos. Após assinatura do Termo de Consentimento Livre e Esclarecido, os participantes foram distribuídos em 2 grupos:

1. Grupo ERRF;

2. Grupo ERT.

Todos os pacientes foram submetidos aos seguintes procedimentos em dois momentos, antes e depois de uma sessão de exercício:

- TA para mensurar VOP, PDA, Alx, Alx75, PP;

- Avaliação do fluxo sanguíneo muscular em repouso e no exercício isométrico a $30 \%$ da contração voluntária máxima (CVM) pela POV;

- Avaliação da PA e FC em repouso e após 15, 30 e 60 minutos após a sessão de exercício.

\subsection{Aspectos éticos}

O presente estudo foi cadastrado no Clinical Trials www.clinicaltrials.gov - sob o registro: NCT 03272737 e foi aprovado pelo Comitê de Ética em Pesquisa do Hospital Israelita Albert Einstein, CAAE: 56798316.4.0000.0071

\subsection{Critério de inclusão}

Ambos os gêneros são elegíveis.

Idosos $\geq 65$ anos com velocidade de marcha $<0,9 \mathrm{~m} / \mathrm{s}^{(71)}$

Assinatura do Termo de Consentimento Livre e Esclarecido. 


\subsection{Critérios de exclusão}

Avaliação médica foi realizada para a inclusão no protocolo, ${ }^{(72)}$ quando os critérios de exclusão abaixo foram verificados:

- Idosos com diabetes mellitus não controlada ou neuropatia periférica;

- Doença arterial obstrutiva periférica sintomática ou índice tornozelo braquial $<0,9$;

- Hipertensão arterial não controlada (PA>160/100mmHg);

- Dislipidemia não controlada (colesterol total >220mg/dL);

- Quadros infecciosos há menos de 1 mês;

- Problemas osteoarticulares ou neurológicos que impedissem a realização do treinamento;

- Histórico de anemia, doença cerebral vascular, infarto do miocárdio nos últimos 6 meses;

- Trombose venosa profunda prévia;

- Embolia pulmonar;

- Uso de anticoagulante oral;

- Uso de dupla antiagregação plaquetária;

- Tabagismo $<6$ meses.

\subsection{Protocolo agudo: sessão de exercício resistido}

Esse estudo experimental faz parte de um projeto maior e ainda em andamento para verificar o efeito do treinamento resistido de baixa intensidade com restrição do fluxo sanguíneo em idosos com baixa velocidade de marcha, para o qual foi calculado um $\mathrm{N}$ amostral de 26 participantes. Sendo assim, nesse estudo experimental nossa amostra é de conveniência, coletada até o momento. Antes da sessão de coleta das variáveis do estudo, os participantes foram submetidos a duas sessões de familiarização e testes de $1 \mathrm{RM}$ destinadas a padronizar a intensidade do exercício de força (Figura 1). 
Programação de atividades da sessão de exercicio resistido

(Duração: 2 semanas / 1 sessão)

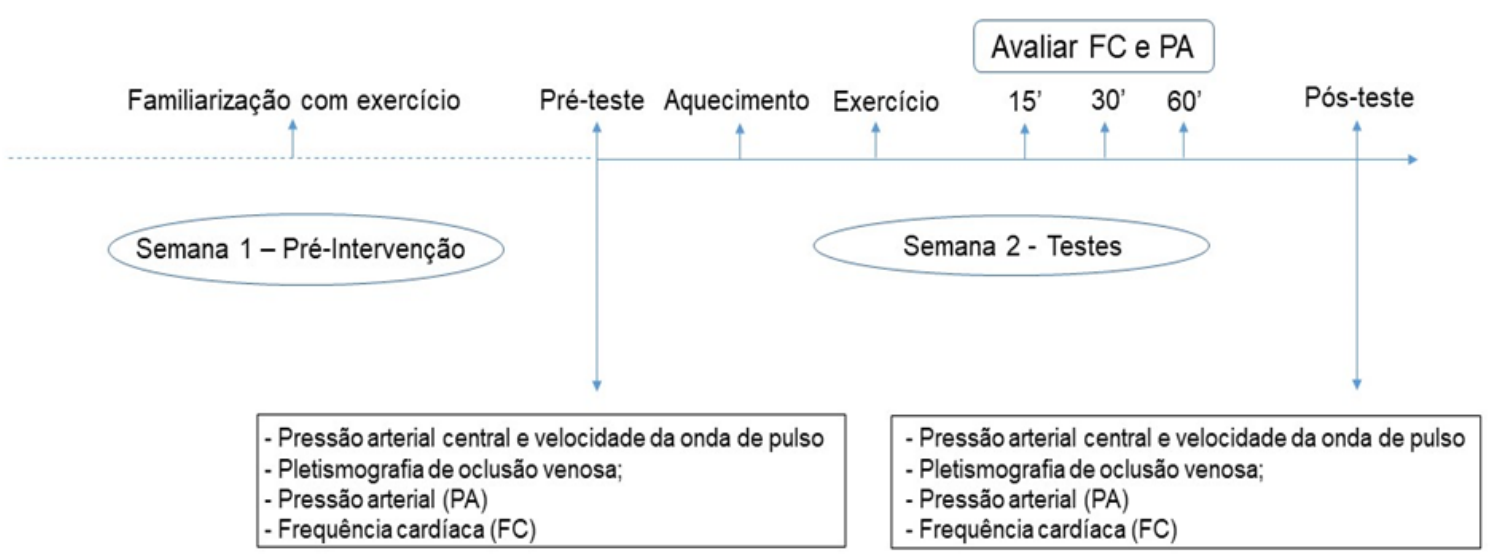

Figura 1. Organograma da sequência de atividades desempenhadas ao longo do estudo agudo

Dentro do laboratório de pesquisa, todos os participantes permaneceram em repouso na posição decúbito dorsal, em uma sala escura descansando quietamente durante 15 minutos antes e depois da sessão de exercício. A temperatura do ambiente foi padronizada entre $20-25^{\circ} \mathrm{C}$ durante todas as metodologias realizadas. Foi recomendado $48 \mathrm{~h}$ antes das sessões de familiarização e da sessão aguda de exercício resistido evitar consumo de bebidas alcoólicas e atividade física. Além disso, 24 horas antes da sessão de exercício foi orientado evitar consumo de produtos com cafeína (ex.: chocolate, café e chá).

Os participantes realizaram 3 séries de 15 repetições com 60 segundos de descanso entre as séries, com a carga mínima permitida na cadeira extensora e no leg press sentado, com intervalo de 72 horas de descanso entre as sessões. No grupo ERRF, foram determinadas pressões apropriadas para que os participantes não tivessem sensação de dor ou incômodo durante a execução do aquecimento e no exercício resistido.

$\mathrm{Na}$ segunda semana, foi realizada uma sessão de exercício resistido com os parâmetros determinados previamente na familiarização. Antes e após esta sessão os idosos foram submetidos a avaliação da rigidez arterial e fluxo sanguíneo muscular. Além disso, após 15, 30 e 60 minutos da execução da sessão, PA e FC foram mensurados pelo aparelho (Dixtal DX 2020 - USA) automaticamente. Além disso, os participantes receberam um lanche padronizado, com baixo teor de gordura. 
$\mathrm{Na}$ tabela 1, estão as informações referentes ao protocolo que foi utilizado na sessão aguda do exercício resistido. Esse protocolo foi baseado no estudo que avaliou o treinamento com restrição moderada do fluxo sanguíneo em pacientes com doenças metabólicas. ${ }^{(56)}$

Tabela 1. Protocolo da sessão aguda de exercício resistido

\begin{tabular}{cccccccc}
\hline Sessões & Grupos & № séries & № repetições & $\begin{array}{c}\text { 1RM } \\
\text { (\%) }\end{array}$ & $\begin{array}{c}\text { CM } \\
(\mathbf{s})\end{array}$ & $\begin{array}{c}\text { Intervalo } \\
(\mathbf{s})\end{array}$ & $\begin{array}{c}\text { TM } \\
(\mathbf{c m})\end{array}$ \\
\hline & ERT & 3 & 15 & 60 & $1-1$ & 60 & $*$ \\
& & & & & & & \\
& ERRF & 3 & 15 & 20 & $1-1$ & 20 & 5 \\
\hline
\end{tabular}

ERT: exercício resistido tradicional; ERRF: exercício com restrição moderada do fluxo sanguíneo; TM: tamanho do manguito; CM: cadência do movimento.

Para testar a segurança da técnica de restrição moderada do fluxo sanguíneo nos idosos na sessão de exercício resistido, um questionário foi preparado para ser respondido durante o estudo agudo pelos participantes. Em função das respostas dos participantes na sessão, a mesma poderia ser interrompida. ${ }^{(72)} \mathrm{O}$ grupo que realizou exercício resistido tradicional também respondeu ao questionário de segurança.

\subsubsection{Teste de 1RM na cadeira extensora e leg press sentado}

A força dinâmica dos membros inferiores foi avaliada pelo teste de repetição máxima de extensão de joelhos na cadeira extensora e no leg press sentado, segundo protocolo apresentado em estudo anterior. ${ }^{(73)}$ Para realização do teste, o indivíduo foi posicionado no aparelho, ajustando as regulagens para evitar desconforto e uso de movimentos adicionais. Os idosos realizaram um aquecimento prévio que consiste em 1 série com 10 repetições com a carga mínima do aparelho. Após o aquecimento específico, os idosos foram submetidos ao teste, realizando uma extensão completa de joelhos, começando o movimento $90^{\circ}$ de flexão e retornando posteriormente a posição inicial. O peso foi progressivamente elevado até que a carga máxima fosse alcançada, com limite máximo de 5 tentativas e intervalo de 3 a 5 minutos entre as mesmas. O teste foi conduzido por um profissional de educação física que incentivou verbalmente os participantes ao longo dessas etapas. 


\subsubsection{Avaliação antropométrica}

O peso corporal foi avaliado por meio da balança da marca Filizola, com precisão de $0,1 \mathrm{~kg}$. A estatura foi obtida por meio do estadiômetro acoplado à balança Filizola, com precisão de $0,5 \mathrm{~cm}$. O IMC foi calculado a partir da relação do peso pela estatura ao quadrado. Para as medidas de circunferência do quadríceps, foi utilizada fita métrica da marca Seca, com precisão de $0,1 \mathrm{~cm}$. As medidas antropométricas foram feitas seguindo a padronização da Sociedade Internacional para Avaliações Antropométricas. ${ }^{(74)}$

\subsubsection{Determinação da pressão da restrição do fluxo sanguíneo}

Nosso estudo foi conduzido pelo aparelho KAATSU Nano (Plaza, Tokyo) que faz automaticamente a restrição do fluxo sanguíneo nos participantes (Figura 2). As pressões de base e ideais de treinamento foram personalizadas para cada participante por um especialista na metodologia que conduziu todos os testes necessários para o protocolo agudo. ${ }^{(75)}$ Os manguitos utilizados tem 5 centímetros de largura e são todos valvulados internamente, garantindo maior conforto para os participantes.

Além disso, o tempo de capilaridade sanguínea estava entre 2 e 3 segundos durante a execução do exercício e foi analisado no quadríceps nos membros inferiores e na palma da mão para membros superiores. Os participantes permaneceram com os manguitos do início ao final da sessão de exercício. 


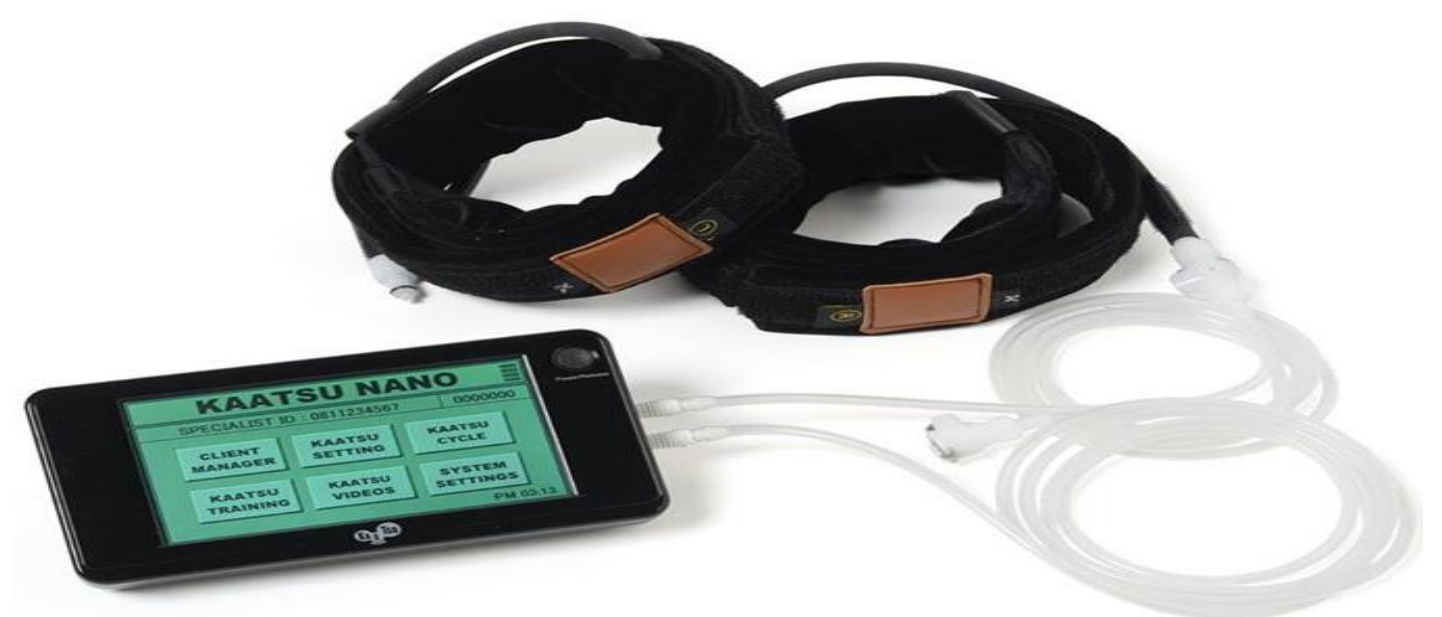

Figura 2. KAATSU Nano e acessórios

\subsubsection{Avaliação do fluxo sanguíneo muscular}

O fluxo sanguíneo muscular foi avaliado pela técnica de pletismografia de oclusão venosa (Figura 3). A perna foi elevada acima do nível do coração para garantir uma adequada drenagem venosa. Um cordão silástico preenchido com mercúrio, conectado a um transdutor de baixa pressão e a um pletismógrafo (D.E. Hokanson), foi colocado ao redor da maior circunferência da região do gastrocnêmio. Um manguito foi colocado ao redor do tornozelo e outro na região distal da coxa. O manguito do tornozelo foi inflado a um nível supra sistólico 30 segundos antes de se iniciarem as medidas. Em intervalos de 15 segundos, o manguito da região distal da coxa foi inflado acima da pressão venosa por um período de sete a oito segundos. O aumento da tensão no cordão silástico refletiu o aumento de volume da perna e, consequentemente, sua vasodilatação. O sinal da onda de fluxo sanguíneo muscular foi registrado em um polígrafo e analisado a cada minuto, realizando-se a média de três registros por minuto, expresso em $\mathrm{mL} / \mathrm{min} / 100 \mathrm{~mL}^{-1}{ }^{(76,77)} \mathrm{O}$ protocolo foi realizado durante 5 minutos de repouso, 3 minutos de exercício isométrico a $30 \%$ da contração voluntária máxima de preensão palmar no dinamômetro de bulbo (Saehan Corp.) e 2 minutos de recuperação. Durante todo o protocolo foram registrados simultaneamente o fluxo sanguíneo muscular, a pressão arterial e frequência cardíaca dos pacientes. A condutância vascular foi calculada pela razão entre o fluxo sanguíneo muscular no membro inferior e a pressão arterial média $(\mathrm{mmHg})$, multiplicado por 100 e expressa em unidades arbitrárias. 


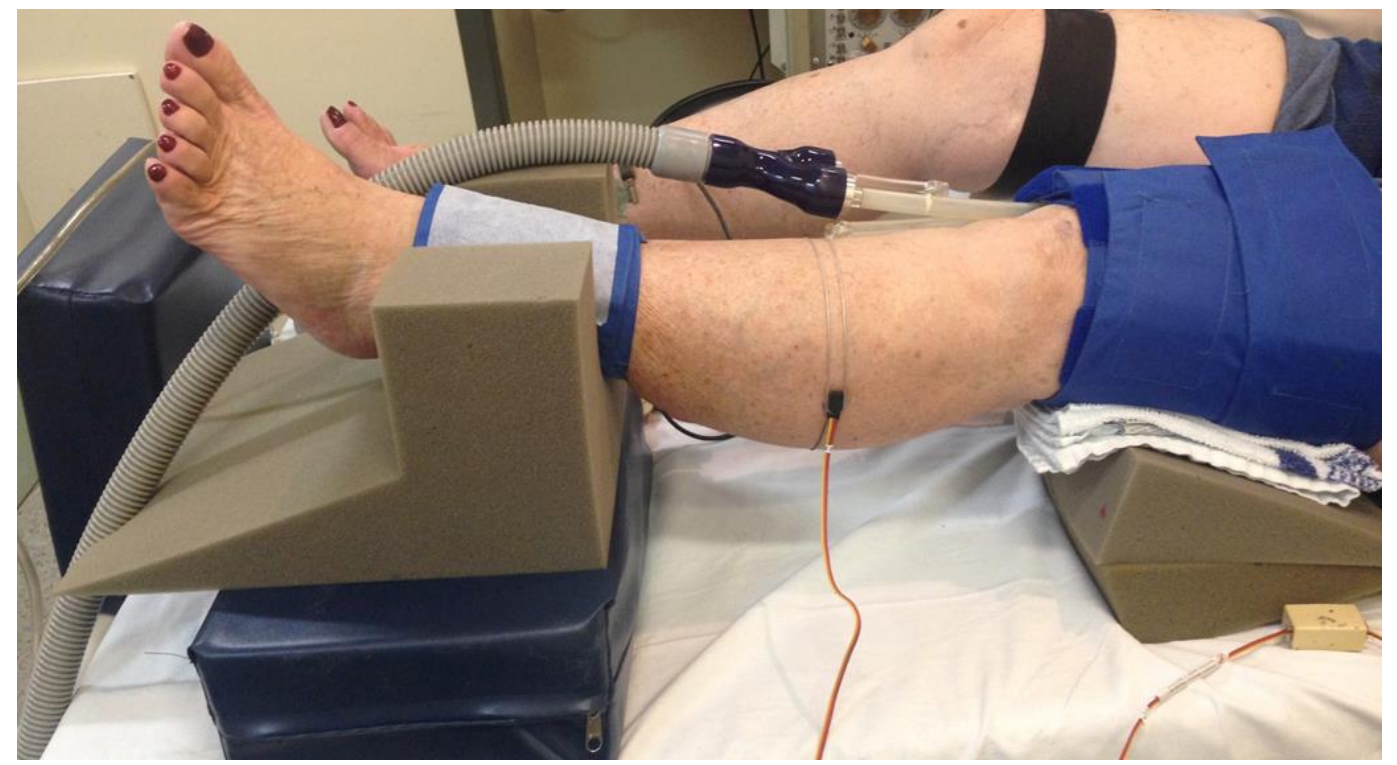

Figura 3. Pletismografia de oclusão venosa

\subsubsection{Medida da rigidez arterial - pressão arterial central e velocidade da onda de pulso}

A pressão arterial central (PAC) foi mensurada pela onda de pulso da artéria radial por meio da técnica de TA (Figura 4). Quando o tonômetro captura uma onda consistente durante 11 segundos, automaticamente os valores obtidos na PAC são armazenados e analisados pelo software (SphygmoCor, AtCor Medical, Austrália).

A estimativa da rigidez arterial foi feita a partir da avaliação da VOP. As ondas de pulso aórtico carótido-femoral foram registradas pela função generalizada de transferência, posicionados acima das artérias carótida e femoral direita, utilizandose um tonômetro.

O registro eletrocardiográfico foi obtido simultaneamente às medidas de onda de pulso aórtico carótido-femoral como referência para calcular o tempo de trânsito da onda. Duas distâncias de superfícies foram medidas pelo investigador: uma entre o ponto de gravação da artéria carótida e da fúrcula esternal (distância 1) e a outra entre a fúrcula esternal e o ponto de gravação na artéria femoral (distância $2) .(78,79)$

As medidas da superfície corporal são usadas para determinar a distância percorrida pela onda de pulso entre os dois pontos de gravação. Sendo 
assim, a distância é dividida pelo tempo de trânsito do pulso, resultando na VOP. As medições são realizadas 3 vezes e a média é utilizada para análise dos dados.

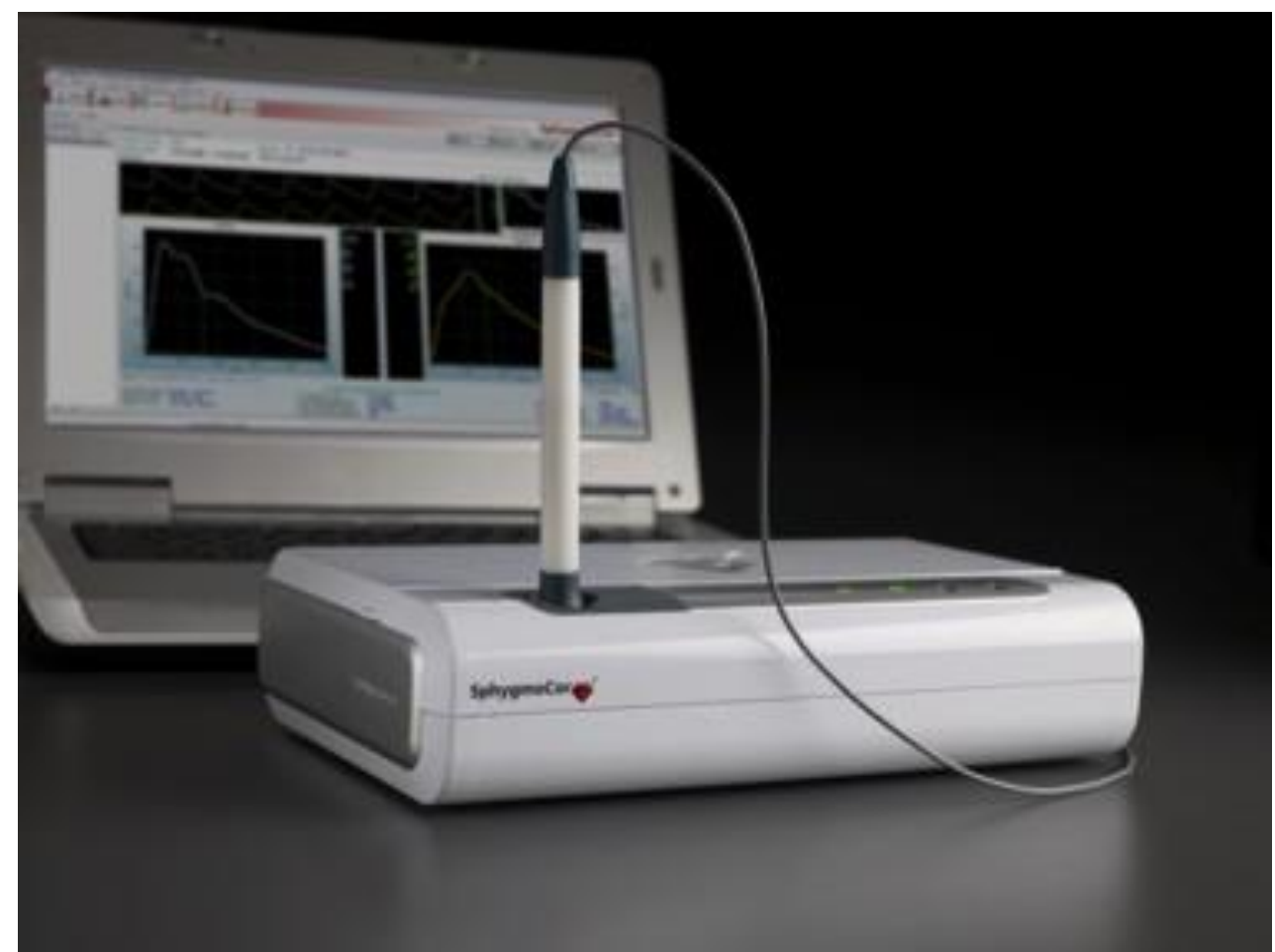

Figura 4. Pressão arterial central e velocidade da onda de pulso

\subsubsection{Análise estatística}

As análises foram realizadas com o auxílio do pacote estatístico SPSS. Para todos os testes foi considerado o nível de significância usual de $5 \%$. Os valores obtidos nos diferentes momentos nos dois grupos são apresentados por medidas resumo como médias, desvios padrão, medianas, mínimo e máximo.

As comparações dos grupos de estudo quanto às medidas observadas no momento inicial foram feitas por testes exatos de Fisher para as variáveis qualitativas e por testes $t$ para as variáveis quantitativas. Para as medidas observadas em mais de um momento, utilizamos modelos de equação de estimação generalizada, considerando o tempo como fator repetido e o grupo de estudo como fator independente. As distribuições foram consideradas de acordo com a adequação à amostra e os resultados foram apresentados por médias estimadas, intervalos de 95\% de confiança e valores-p para as comparações entre grupos e momentos. 


\section{RESULTADOS}

Os resultados do estudo demonstraram que uma sessão aguda de exercício resistido associado a restrição do fluxo promoveu aumento significativo nos indicadores indiretos da rigidez arterial - PDA, pressão diastólica radial, pressão diastólica aórtica, Alx e Alx75, o que provavelmente foi associado a uma maior resistência periférica. Entretanto, não houve impacto na velocidade de onda de pulso, fluxo sanguíneo muscular, condutância vascular e dos parâmetros hemodinâmicos avaliados até 1 hora após a sessão.

Foram avaliados no estudo 17 participantes, sendo 7 no grupo controle e 10 do grupo intervenção, com idade entre 70 e 89 anos, sendo a maioria do sexo feminino. Não observamos evidências de diferenças significativas entre os grupos de intervenção em relação às características físicas e dados basais dos participantes ( $p>0,05$ em todas as comparações) (Tabela 2).

Tabela 2. Características físicas e dados basais dos participantes

\begin{tabular}{|c|c|c|c|c|}
\hline \multirow[b]{2}{*}{ Características } & \multirow[b]{2}{*}{$\begin{array}{l}\text { Total } \\
(n=17)\end{array}$} & \multicolumn{2}{|c|}{ Grupo de Intervenção } & \multirow[b]{2}{*}{ Valor-p } \\
\hline & & $\begin{array}{l}\text { ERT } \\
(n=7)\end{array}$ & $\begin{array}{l}\text { ERRF } \\
(n=10)\end{array}$ & \\
\hline Gênero (\%) & & & & $>0,999^{\#}$ \\
\hline Feminino & $14(82,4)$ & $6(85,7)$ & $8(80,0)$ & \\
\hline Masculino & $3(17,6)$ & $1(14,3)$ & $2(20,0)$ & \\
\hline Idade (anos) & & & & $0,796^{\$}$ \\
\hline Média (DP) & $82,5(6,0)$ & $82,0(7,4)$ & $82,8(5,2)$ & \\
\hline Mediana $\left(1^{\circ} \mathrm{Q}\right.$; $\left.3^{\circ} \mathrm{O} \mathrm{Q}\right)$ & $84(81 ; 87)$ & $83(74 ; 88)$ & $84(81 ; 86)$ & \\
\hline Mínimo; Máximo & $70 ; 89$ & $70 ; 89$ & $71 ; 89$ & \\
\hline Velocidade de marcha $(\mathrm{m} / \mathrm{s})$ & & & & 0,052 \\
\hline Média (DP) & $0,66(0,13)$ & $0,74(0,11)$ & $0,61(0,13)$ & \\
\hline Mediana $\left(1^{\circ} \mathrm{Q} ; 3^{\circ} \mathrm{Q}\right)$ & $0,68(0,57 ; 0,73)$ & $0,73(0,69 ; 0,86)$ & $0,59(0,54 ; 0,68)$ & \\
\hline Mínimo; Máximo & 0,$39 ; 0,87$ & 0,$54 ; 0,87$ & 0,$39 ; 0,86$ & \\
\hline Altura $(m)$ & & & & $0,774^{\$}$ \\
\hline Média (DP) & $1,55(0,07)$ & $1,54(0,06)$ & $1,55(0,07)$ & \\
\hline Mediana (1ำ; $\left.3^{\circ} \mathrm{Q}\right)$ & $1,54(1,52 ; 1,62)$ & $1,54(1,50 ; 1,62)$ & $1,53(1,52 ; 1,62)$ & \\
\hline Mínimo; Máximo & 1,$46 ; 1,68$ & 1,$46 ; 1,62$ & 1,$47 ; 1,68$ & \\
\hline IMC $(\mathrm{Kg})$ & & & & $0,762^{\$}$ \\
\hline Média (DP) & $28,1(4,9)$ & $28,5(6,3)$ & $27,8(3,9)$ & \\
\hline Mediana (1ํㅜ; $\left.3^{\circ} \mathrm{Q}\right)$ & $27,7(24,8 ; 31,3)$ & $27,7(24,0 ; 32,4)$ & $27,8(24,8 ; 31,3)$ & \\
\hline Mínimo; Máximo & 19,$5 ; 39,3$ & 19,$5 ; 39,3$ & 21,$4 ; 32,7$ & \\
\hline
\end{tabular}

$\mathrm{Na}$ tabela 3 estão descritas as comorbidades e fatores de riscos associados aos participantes do estudo. As comorbidades prévias mais observadas em 
nossa amostra foram dislipidemia $(70,6 \%)$ e hipertensão arterial sistêmica $(88,2 \%)$ (Tabela 3). Não observamos evidências de diferença estatisticamente significante entre os grupos de intervenção em relação à presença das comorbidades ( $p>0,05$ em todas as comparações).

Tabela 3. Comorbidades e doenças associadas aos participantes do estudo

\begin{tabular}{|c|c|c|c|c|c|c|c|}
\hline \multirow{2}{*}{$\begin{array}{l}\text { Comorbidades e } \\
\text { doenças }\end{array}$} & \multirow[b]{2}{*}{ ERT (7) } & \multicolumn{2}{|c|}{ Grupos } & \multirow[b]{2}{*}{$\%$} & \multirow{2}{*}{ Total } & \multirow{2}{*}{$\%$} & \multirow{2}{*}{ Valor-p } \\
\hline & & $\%$ & ERRF (10) & & & & \\
\hline Obesidade & & & & & & & 0,593 \\
\hline Não & 4 & 57,1 & 8 & 80,0 & 12 & 70,6 & \\
\hline Sim & 3 & 42,9 & 2 & 20,0 & 5 & 29,4 & \\
\hline Depressão & & & & & & & $>0,999$ \\
\hline Não & 5 & 71,4 & 6 & 60,0 & 11 & 64,7 & \\
\hline Sim & 2 & 28,6 & 4 & 40,0 & 6 & 35,3 & \\
\hline $\begin{array}{l}\text { Acidente vascular } \\
\text { cerebral }\end{array}$ & & & & & & & $>0,999$ \\
\hline Não & 7 & 100,0 & 9 & 90,0 & 16 & 94,1 & \\
\hline Sim & 0 & 0,0 & 1 & 10,0 & 1 & 5,9 & \\
\hline $\begin{array}{l}\text { Infarto agudo do } \\
\text { miocárdio }\end{array}$ & & & & & & & $>0,999$ \\
\hline Não & 5 & 71,4 & 8 & 80,0 & 13 & 76,5 & \\
\hline Sim & 2 & 28,6 & 2 & 20,0 & 4 & 23,5 & \\
\hline Mal de Parkinson & & & & & & & 0,485 \\
\hline Não & 7 & 100,0 & 8 & 80,0 & 15 & 88,2 & \\
\hline Sim & 0 & 0,0 & 2 & 20,0 & 2 & 11,8 & \\
\hline Demência & & & & & & & $>0,999$ \\
\hline Não & 6 & 85,7 & 9 & 90,0 & 15 & 88,2 & \\
\hline Sim & 1 & 14,3 & 1 & 10,0 & 2 & 11,8 & \\
\hline Diabetes & & & & & & & 0,537 \\
\hline Não & 5 & 71,4 & 9 & 90,0 & 14 & 82,4 & \\
\hline Sim & 2 & 28,6 & 1 & 10,0 & 3 & 17,6 & \\
\hline Fraturas & & & & & & & 0,412 \\
\hline Não & 6 & 85,7 & 10 & 100,0 & 16 & 94,1 & \\
\hline Sim & 1 & 14,3 & 0 & 0,0 & 1 & 5,9 & \\
\hline Osteoporose & & & & & & & $>0,999$ \\
\hline Não & 6 & 85,7 & 9 & 90,0 & 15 & 88,2 & \\
\hline Sim & 1 & 14,3 & 1 & 10,0 & 2 & 11,8 & \\
\hline Osteoartrose & & & & & & & $>0,999$ \\
\hline Não & 5 & 71,4 & 7 & 70,0 & 12 & 70,6 & \\
\hline Sim & 2 & 28,6 & 3 & 30,0 & 5 & 29,4 & \\
\hline Dislipidemia & & & & & & & 0,593 \\
\hline Não & 3 & 42,9 & 2 & 20,0 & 5 & 29,4 & \\
\hline Sim & 4 & 57,1 & 8 & 80,0 & 12 & 70,6 & \\
\hline $\begin{array}{l}\text { Hipertensão arterial } \\
\text { sistêmica }\end{array}$ & & & & & & & 0,154 \\
\hline Não & 2 & 28,6 & 0 & 0,0 & 2 & 11,8 & \\
\hline Sim & 5 & 71,4 & 10 & 100,0 & 15 & 88,2 & \\
\hline
\end{tabular}

Valores expressos por médias estimadas. ERT: exercício resistido tradicional; ERRF: exercício resistido com moderada restrição do fluxo sanguíneo.

Em relação ao uso de medicações prévias (Tabela 4), quinze $(88,2 \%)$ idosos usavam anti-hipertensivos, dez $(58,8 \%)$ utilizavam medicamentos para dislipidemia, nove $(52,9 \%)$ antidepressivos, além de outros medicamentos. Não 
observamos diferença estatisticamente significante entre os grupos de intervenção em relação ao uso de diferentes medicações ( $p>0,05$ em todas as comparações).

Tabela 4. Medicações dos participantes

\begin{tabular}{|c|c|c|c|c|c|c|c|}
\hline Medicações & ERT & $n=7$ & ERRF & $\mathrm{n}=10$ & Total & $\%$ & Valor-p \\
\hline Anti-hipertensivo & & & & & & & 0,154 \\
\hline Não & 2 & 28,6 & 0 & 0,0 & 2 & 11,8 & \\
\hline Sim & 5 & 71,4 & 10 & 100,0 & 15 & 88,2 & \\
\hline Dislipidemia & & & & & & & 0,350 \\
\hline Não & 4 & 57,1 & 3 & 30,0 & 7 & 41,2 & \\
\hline Sim & 3 & 42,9 & 7 & 70,0 & 10 & 58,8 & \\
\hline Antidepressivo & & & & & & & 0,637 \\
\hline Não & 4 & 57,1 & 4 & 40,0 & 8 & 47,1 & \\
\hline Sim & 3 & 42,9 & 6 & 60,0 & 9 & 52,9 & \\
\hline Antiparkinsoniano & & & & & & & 0,485 \\
\hline Não & 7 & 100,0 & 8 & 80,0 & 15 & 88,2 & \\
\hline Sim & 0 & 0,0 & 2 & 20,0 & 2 & 11,8 & \\
\hline Ansiolítico & & & & & & & 0,485 \\
\hline Não & 7 & 100,0 & 8 & 80,0 & 15 & 88,2 & \\
\hline Sim & 0 & 0,0 & 2 & 20,0 & 2 & 11,8 & \\
\hline Anticonvulsivo & & & & & & & $>0,999$ \\
\hline Não & 6 & 85,7 & 9 & 90,0 & 15 & 88,2 & \\
\hline Sim & 1 & 14,3 & 1 & 10,0 & 2 & 11,8 & \\
\hline Hipoglicemiantes orais & & & & & & & 0,101 \\
\hline Não & 3 & 42,9 & 9 & 90,0 & 12 & 70,6 & \\
\hline Sim & 4 & 57,1 & 1 & 10,0 & 5 & 29,4 & \\
\hline Antipsicótico & & & & & & & $>0,999$ \\
\hline Não & 6 & 85,7 & 9 & 90,0 & 15 & 88,2 & \\
\hline Sim & 1 & 14,3 & 1 & 10,0 & 2 & 11,8 & \\
\hline $\begin{array}{l}\text { Medicação para } \\
\text { demência }\end{array}$ & & & & & & & $>0,999$ \\
\hline Não & 6 & 85,7 & 9 & 90,0 & 15 & 88,2 & \\
\hline Sim & 1 & 14,3 & 1 & 10,0 & 2 & 11,8 & \\
\hline $\begin{array}{l}\text { Antiagregante } \\
\text { plaquetário }\end{array}$ & & & & & & & 0,644 \\
\hline Não & 4 & 57,1 & 7 & 70,0 & 11 & 64,7 & \\
\hline $\mathrm{Sim}$ & 3 & 42,9 & 3 & 30,0 & 6 & 35,3 & \\
\hline Outras & & & & & & & $>0,999$ \\
\hline Não & 2 & 28,6 & 2 & 20,0 & 4 & 23,5 & \\
\hline Sim & 5 & 71,4 & 8 & 80,0 & 13 & 76,5 & \\
\hline
\end{tabular}

$\mathrm{Na}$ tabela 5 são expostas as respostas da pressão arterial central e variáveis dos índices de rigidez arterial nos períodos pré e pós-intervenção em ambos os grupos. Não observamos evidências de diferenças significativas dessas variáveis no momento pré-intervenção ( $p>0,05$ em todas as comparações).

Apenas o grupo ERRF demonstrou aumento significativo no momento pós intervenção nos indicadores PP aórtica, $(p=0,033)$ para pressão arterial sistólica (PAS) radial, $(0,040)$, PP radial $(0,045)$ e Alx $(p=0,042)$ e pressão de aumentação (PDA) $(0,003)$. 
No momento pós-intervenção as médias do grupo ERRF foram maiores do que as do grupo ERT para pressão arterial diastólica (PAD) aórtica $(p=0,029)$, para a PDA $(p=0,026)$, PAD radial $(p=0,017)$, para o Alx $(p=0,008)$ e Alx75 $(p=0,014)$.

Tabela 5. Indicadores da pressão arterial central e velocidade da onda de pulso

\begin{tabular}{|c|c|c|c|}
\hline \multirow{2}{*}{$\begin{array}{l}\text { Tonometria de } \\
\text { aplanação }\end{array}$} & \multirow[b]{2}{*}{ Pré-intervenção } & Momento & \multirow{2}{*}{ Valor-p } \\
\hline & & Pós-intervenção & \\
\hline \multicolumn{4}{|c|}{ PAS aórtica $(\mathrm{mmHg})$} \\
\hline Grupo ERT & $118,1(105,6 ; 130,7)$ & $124,4(110,3 ; 138,6)$ & 0,196 \\
\hline Grupo ERRF & $126,0(115,0 ; 137,0)$ & $135,6(122,3 ; 148,8)$ & 0,053 \\
\hline Valor-p $\$$ & 0,343 & 0,249 & \\
\hline \multicolumn{4}{|c|}{ PAD aórtica $(\mathrm{mmHg})$} \\
\hline Grupo ERT & $63,0(57,5 ; 68,5)$ & $61,7(56,0 ; 67,5)$ & 0,498 \\
\hline Grupo ERRF & $69,4(64,6 ; 74,3)$ & $70,5(65,2 ; 75,9)$ & 0,557 \\
\hline Valor-p $\$$ & 0,081 & 0,029 & \\
\hline \multicolumn{4}{|c|}{ PAM aórtica $(\mathrm{mmHg})$} \\
\hline Grupo ERT & $84,9(76,9 ; 92,8)$ & $86,0(77,0 ; 95,0)$ & 0,697 \\
\hline Grupo ERRF & $91,8(84,8 ; 98,8)$ & $96,2(87,7 ; 104,6)$ & 0,142 \\
\hline Valor-p $p^{\$}$ & 0,189 & 0,102 & \\
\hline \multicolumn{4}{|c|}{ PP aórtica $(\mathrm{mmHg})$} \\
\hline Grupo ERT & $55,1(45,6 ; 64,7)$ & $62,7(51,9 ; 73,5)$ & 0,075 \\
\hline Grupo ERRF & $56,6(48,1 ; 65,0)$ & $65,7(55,4 ; 75,9)$ & 0,033 \\
\hline Valor-p $\mathrm{p}^{\Phi}$ & 0,821 & 0,687 & \\
\hline \multicolumn{4}{|c|}{ PAS radial $(\mathrm{mmHg})$} \\
\hline Grupo ERT & $129,1(116,5 ; 141,7)$ & $135,7(121,2 ; 150,2)$ & 0,157 \\
\hline Grupo ERRF & $133,2(122,1 ; 144,3)$ & $143,0(129,4 ; 156,5)$ & 0,040 \\
\hline Valor-p $\$$ & 0,622 & 0,459 & \\
\hline \multicolumn{4}{|c|}{ PAD radial $(\mathrm{mmHg})$} \\
\hline Grupo ERT & $62,3(57,0 ; 67,5)$ & $60,6(55,0 ; 66,1)$ & 0,352 \\
\hline Grupo ERRF & $68,8(64,1 ; 73,4)$ & $70,0(64,8 ; 75,2)$ & 0,508 \\
\hline Valor-p $\mathrm{p}^{\$}$ & 0,068 & 0,017 & \\
\hline \multicolumn{4}{|c|}{ PAM radial $(\mathrm{mmHg})$} \\
\hline Grupo ERT & $84,9(76,9 ; 92,8)$ & $86,0(77,0 ; 95,0)$ & 0,697 \\
\hline Grupo ERRF & $91,8(84,8 ; 98,8)$ & $96,2(87,7 ; 104,6)$ & 0,142 \\
\hline Valor- $\mathrm{p}^{\$}$ & 0,189 & 0,102 & \\
\hline \multicolumn{4}{|l|}{ PP radial $(\mathrm{mmHg})$} \\
\hline Grupo ERT & $66,9(56,9 ; 76,9)$ & $75,1(63,4 ; 86,8)$ & 0,069 \\
\hline Grupo ERRF & $64,4(55,6 ; 73,3)$ & $73,6(62,5 ; 84,7)$ & 0,045 \\
\hline \multicolumn{4}{|l|}{ PDA } \\
\hline Grupo ERT & $19,9(13,3 ; 26,4)$ & $22,9(16,3 ; 29,4)$ & 0,313 \\
\hline Grupo ERRF & $23,9(18,1 ; 29,7)$ & $33,2(27,0 ; 39,5)$ & 0,003 \\
\hline valor-p ${ }^{\$}$ & 0,352 & 0,026 & \\
\hline Valor-p $\mathrm{p}^{\$}$ & 0,618 & 0,512 & \\
\hline \multicolumn{4}{|c|}{ Carótida/femoral (m/s) } \\
\hline Grupo ERT & $8,4(6,9 ; 10,1)$ & $7,7(6,4 ; 9,3)$ & 0,493 \\
\hline Grupo ERRF & $7,7(6,5 ; 9,2)$ & $7,9(6,8 ; 9,2)$ & 0,791 \\
\hline Valor-p $p^{\$}$ & 0,509 & 0,831 & \\
\hline \multicolumn{4}{|l|}{ Alx $(\%)$} \\
\hline Grupo ERT & $36,3(27,6 ; 45,0)$ & $36,4(29,4 ; 43,5)$ & 0,973 \\
\hline Grupo ERRF & $41,9(34,2 ; 49,6)$ & $50,2(43,3 ; 57,1)$ & 0,042 \\
\hline Valor-p $\$$ & 0,330 & 0,008 & \\
\hline Alx75 (\%) & & & \\
\hline
\end{tabular}




\begin{tabular}{|c|c|c|c|}
\hline Grupo ERT & $31,9(21,9 ; 41,8)$ & $31,3(24,0 ; 38,5)$ & 0,898 \\
\hline Grupo ERRF & $35,9(27,1 ; 44,7)$ & $44,3(37,2 ; 51,3)$ & 0,054 \\
\hline \multicolumn{4}{|c|}{$\begin{array}{l}\text { Velocidade de onda de } \\
\text { pulso pré-treinamento } \\
\text { agudo }(\mathrm{m} / \mathrm{s})\end{array}$} \\
\hline Grupo ERT & $11,6(8,7 ; 15,5)$ & $12,7(9,6 ; 16,8)$ & 0,091 \\
\hline Grupo ERRF & $10,6(8,1 ; 13,8)$ & $11,2(8,7 ; 14,5)$ & 0,157 \\
\hline Valor-p $\$$ & 0,618 & 0,512 & \\
\hline \multicolumn{4}{|c|}{$\begin{array}{l}\text { Valores expressos por médias estimadas e desvio padrão; \# negrito: pré x pós-intervenção } \\
\text { para cada grupo; \$ negrito: Grupo ERT x Grupo ERRF em cada momento. ERT: Exercício } \\
\text { resistido tradicional; ERRF: exercício resistido com moderada restrição do fluxo sanguíneo. } \\
\text { PAS: pressão arterial sistólica; PAD: pressão arterial diastólica; PAM: pressão arterial média; } \\
\text { PP: pressão de pulso; PDA: pressão de aumentação; VOP: velocidade da onda de pulso; Alx } \\
\text { índice de aumentação; Alx75: Índice de aumentação corrigido à } 75 \mathrm{bpm} \text {. }\end{array}$} \\
\hline
\end{tabular}

Comparamos os grupos em relação às médias de fluxo sanguíneo muscular, condutância vascular, FC, PAS, PAD e pressão arterial média (PAM) em repouso nos momentos pré e pós-intervenção (Tabela 6) e não observamos evidências de diferenças significativas nos dois momentos ( $p>0,05$ em todas as comparações) (Tabela 6).

Tabela 6. Medidas em repouso obtidas na pletismografia de oclusão venosa

\begin{tabular}{|c|c|c|c|}
\hline \multirow{2}{*}{ Média em repouso } & \multicolumn{2}{|c|}{ Momento } & \multirow{2}{*}{ Valor-p } \\
\hline & Pré-intervenção & Pós-intervenção & \\
\hline \multicolumn{4}{|l|}{$\mathrm{FSM}\left(\mathrm{ml}^{\mathrm{kg}} \mathrm{kg}^{-1} \cdot \mathrm{min}^{-1}\right)$} \\
\hline Grupo ERT & $1,70(1,23 ; 2,17)$ & $1,72(1,25 ; 2,19)$ & 0,947 \\
\hline Grupo ERRF & $1,71(1,30 ; 2,13)$ & $1,78(1,37 ; 2,19)$ & 0,836 \\
\hline Valor-p $\mathrm{p}^{\$}$ & 0.969 & 0,860 & \\
\hline \multicolumn{4}{|c|}{ Frequência cardíaca (bpm) } \\
\hline Grupo ERT & $61,5(52,7 ; 70,2)$ & $63,4(54,6 ; 72,1)$ & 0,148 \\
\hline Grupo ERRF & $64,2(56,8 ; 71,6)$ & $64,4(57,0 ; 71,9)$ & 0,834 \\
\hline Valor-p $p^{\$}$ & 0,623 & 0,847 & \\
\hline \multicolumn{4}{|c|}{ Pressão arterial sistólica (mmHg) } \\
\hline Grupo ERT & $128,4(110,6 ; 146,1)$ & $133,5(118,2 ; 148,8)$ & 0,281 \\
\hline Grupo ERRF & $127,1(112,1 ; 142,1)$ & $134,9(122,0 ; 147,8)$ & 0,060 \\
\hline Valor-p $\$$ & 0,910 & 0,886 & \\
\hline \multicolumn{4}{|c|}{ Pressão arterial diastólica (mmHg) } \\
\hline Grupo ERT & $59,9(53,4 ; 66,4)$ & $63,2(56,3 ; 70,0)$ & 0,217 \\
\hline Grupo ERRF & $63,6(58,2 ; 69,1)$ & $66,4(60,6 ; 72,2)$ & 0,211 \\
\hline Valor-p $\$$ & 0,367 & 0,456 & \\
\hline \multicolumn{4}{|c|}{ Pressão arterial média $(\mathrm{mmHg})$} \\
\hline Grupo ERT & $82,7(72,7 ; 92,7)$ & $86,6(77,9 ; 95,3)$ & 0,210 \\
\hline Grupo ERRF & $84,8(76,3 ; 93,2)$ & $89,3(81,9 ; 96,6)$ & 0,093 \\
\hline Valor-p $\$$ & 0,745 & 0,634 & \\
\hline \multicolumn{4}{|c|}{ Condutância vascular (und) } \\
\hline Grupo ERT & $2,02(1,32 ; 2,73)$ & $2,26(1,67 ; 2,85)$ & 0,520 \\
\hline Grupo ERRF & $2,12(1,52 ; 2,72)$ & $2,02(1,52 ; 2,52)$ & 0,749 \\
\hline Valor-p $\mathrm{p}^{\$}$ & 0,833 & 0,531 & \\
\hline
\end{tabular}


Na tabela 7 são apresentados os dados de comparação entre os grupos para as variáveis da pletismografia de oclusão venosa no período pré e pósintervenção durante a realização do handgrip. Nas comparações entre os momentos pré e pós-intervenção em cada grupo de treinamento não observamos evidências de variações significativas nos picos das variáveis de fluxo sanguíneo muscular, FC, PAS, PAD e condutância vascular durante o handgrip, nos dois grupos ( $p>0,05$ em todas as comparações). Entretanto, o grupo ERRF demonstrou aumento significativo da PAM durante o handgrip no momento pós-intervenção (diferença média: 11,1; IC 95\%: 0,1 a 22,2; $p=0,049)$. Não observamos evidências de diferenças significativas entre os grupos nos dois momentos ( $p>0,05$ em todas as comparações).

Tabela 7. Picos das medidas durante o handgrip obtidas pela pletismografia de oclusão venosa

\begin{tabular}{|c|c|c|c|}
\hline Pico durante o handgrip & \multicolumn{2}{|c|}{ Momento } & Valor-p \\
\hline \multicolumn{4}{|l|}{ FSM (ml. $\left.\mathrm{kg}^{-1} \cdot \mathrm{min}^{-1}\right)$} \\
\hline Grupo ERT & $2,23(1,71 ; 2,76)$ & $2,57(2,12 ; 3,02)$ & 0,316 \\
\hline Grupo ERRF & $1,98(1,52 ; 2,44)$ & $2,32(1,92 ; 2,72)$ & 0,252 \\
\hline Valor-p $\mathrm{p}^{\Phi}$ & 0,459 & 0,403 & \\
\hline \multicolumn{4}{|l|}{ Frequência cardíaca (bpm) } \\
\hline Grupo ERT & $71,6(61,3 ; 81,9)$ & $72,0(62,7 ; 81,3)$ & 0,850 \\
\hline Grupo ERRF & $73,9(65,2 ; 82,5)$ & $76,0(68,1 ; 83,9)$ & 0,238 \\
\hline Valor-p $p^{\$}$ & 0,730 & 0,502 & \\
\hline \multicolumn{4}{|c|}{ Pressão arterial sistólica $(\mathrm{mmHg})$} \\
\hline Grupo ERT & $171,8(149,3 ; 194,3)$ & $166,2(144,2 ; 188,2)$ & 0,533 \\
\hline Grupo ERRF & $159,0(140,0 ; 178,0)$ & $172,0(153,4 ; 190,6)$ & 0,097 \\
\hline Valor-p $\$$ & 0,375 & 0,679 & \\
\hline \multicolumn{4}{|c|}{ Pressão arterial diastólica $(\mathrm{mmHg})$} \\
\hline Grupo ERT & $80,8(70,9 ; 90,7)$ & $82,4(71,5 ; 93,3)$ & 0,791 \\
\hline Grupo EERF & $77,6(69,2 ; 86,0)$ & $87,6(78,4 ; 96,8)$ & 0,061 \\
\hline Valor- $\mathrm{p}^{\$}$ & 0,610 & 0,459 & \\
\hline \multicolumn{4}{|c|}{ Pressão arterial média $(\mathrm{mmHg})$} \\
\hline Grupo ERT & $109,2(97,7 ; 120,7)$ & $108,7(96,0 ; 121,3)$ & 0,933 \\
\hline Grupo ERRF & $102,0(92,2 ; 111,7)$ & $113,1(102,4 ; 123,8)$ & 0,049 \\
\hline Valor-p $\mathrm{p}^{\Phi}$ & 0,328 & 0,583 & \\
\hline \multicolumn{4}{|l|}{ Condutância vascular (Und) } \\
\hline Grupo ERT & $2,17(1,51 ; 2,83)$ & $2,82(2,20 ; 3,43)$ & 0,058 \\
\hline Grupo ERRF & $2,13(1,57 ; 2,69)$ & $2,27(1,72 ; 2,83)$ & 0,615 \\
\hline Valor-p $\mathrm{p}^{\$}$ & 0,916 & 0,186 & \\
\hline
\end{tabular}

Valores expressos por médias estimadas (IC 95\%); \# negrito: pré x pós-intervenção para cada grupo; \$ negrito: Grupo ERT x Grupo ERRF em cada momento. ERT: exercício resistido tradicional; ERRF: exercício resistido com moderada restrição do fluxo sanguíneo. FSM: fluxo sanguíneo muscular.

Avaliamos o comportamento da FC, PAS, PAD e PAM ao longo do tempo em cada grupo de intervenção.

Observamos evidências de aumento da FC nas avaliações de 15, 30 e 60 minutos comparadas ao momento inicial $(p=0,002$ em 15min, $p<0,001$ em 30min e 
$p=0,018$ em 60min) apenas no grupo ERT, mas não evidenciamos diferença significativa entre os grupos $(p>0,05)$.

Não observamos evidências de variações de PAS, PAD e PAM ao longo do tempo em ambos os grupos ( $p>0,05$ em todas as comparações). Entretanto, a PAD demonstrou diferença significativa apenas na avaliação de 60 minutos $(p=0,038)$, entre os grupos, onde observamos maior PAD no grupo ERRF (Tabela 8).

Tabela 8. Variáveis hemodinâmicas pré e pós-sessão aguda de exercício resistido

\begin{tabular}{|c|c|c|c|c|}
\hline Variáveis & & & $\begin{array}{l}\text { mento } \\
\text { nutos) }\end{array}$ & \\
\hline & 0 & 15 & 30 ' & $60^{\prime}$ \\
\hline $\mathrm{FC}$ & & & & \\
\hline Grupo ERT & $63,0(55,9 ; 71,0)$ & $70,6(63,9 ; 78,0)$ & $66,2(59,6 ; 73,5)$ & $66,7(60,0 ; 74,1)$ \\
\hline Valor-p $p^{\#}$ & & 0,002 & $<0,001$ & 0,018 \\
\hline Grupo ERRF & $64,3(57,8 ; 71,5)$ & $65,9(61,2 ; 71,0)$ & $65,3(59,7 ; 71,4)$ & $64,2(58,7 ; 70,3)$ \\
\hline Valor-p $\#$ & & $>0,999$ & $>0,999$ & $>0,999$ \\
\hline Valor-p & 0,802 & 0,282 & 0,844 & 0,588 \\
\hline PAS & & & & \\
\hline Grupo ERT & $145,1(136,9 ; 153,9)$ & $135,0(128,6 ; 141,8)$ & $141,4(130,2 ; 153,7)$ & $132,7(119,1 ; 147,9)$ \\
\hline Valor-p $p^{\#}$ & & 0,233 & 0,468 & 0,114 \\
\hline Grupo ERRF & $135,9(123,9 ; 149,0)$ & $132,3(116,2 ; 150,6)$ & $129,4(112,1 ; 149,4)$ & $135,8(121,3 ; 152,1)$ \\
\hline Valor-p $p^{\#}$ & & 0,878 & 0,724 & 0,984 \\
\hline Valor-p & 0,231 & 0,774 & 0,283 & 0,774 \\
\hline PAD & & & & \\
\hline Grupo ERT & $63,9(57,4 ; 71,1)$ & $70,4(65,2 ; 76,1)$ & $63,4(58,3 ; 69,1)$ & $62,0(56,7 ; 67,8)$ \\
\hline Valor-p $p^{\#}$ & & 0,398 & 0,837 & 0,657 \\
\hline Grupo ERRF & $69,5(64,4 ; 74,9)$ & $67,2(59,9 ; 75,4)$ & $66,5(61,8 ; 71,6)$ & $69,2(65,4 ; 73,2)$ \\
\hline Valor-p $p^{\#}$ & & 0,700 & 0,700 & 0,888 \\
\hline Valor-p & 0,199 & 0,505 & 0,409 & 0,038 \\
\hline PAM & & & & \\
\hline Grupo ERT & $93,9(88,0 ; 100,1)$ & $91,0(85,2 ; 97,2)$ & $95,7(86,8 ; 105,6)$ & $87,9(80,8 ; 95,5)$ \\
\hline Valor-p $p^{\#}$ & & 0,790 & 0,790 & 0,568 \\
\hline Grupo ERRF & $92,0(83,9 ; 100,8)$ & $90,6(78,4 ; 104,7)$ & $88,8(78,5 ; 100,4)$ & $93,8(86,1 ; 102,2)$ \\
\hline Valor-p $p^{\#}$ & & $>0,999$ & $>0,999$ & $>0,999$ \\
\hline Valor-p & 0,726 & 0,957 & 0,346 & 0,284 \\
\hline
\end{tabular}




\section{DISCUSSÃO}

Os participantes incluídos no presente estudo apresentavam baixa velocidade de marcha, idade $\geq 70$ anos, índice de massa corporal $>25$ e diversas comorbidades e fatores de risco associados. A maioria dos participantes possuíam hipertensão arterial sistêmica (88,2\%) e dislipidemia $(70,6 \%)$, o que por si só, é associado a outras comorbidades, causa modificações no sistema vascular, como aumento na rigidez arterial e redução do fluxo sanguíneo muscular.

Enquanto a VOP em indivíduos jovens e saudáveis em um estudo prévio era em torno de 3 a $5 \mathrm{~m} / \mathrm{s},{ }^{(33)}$ em idosos $\geq 70$ anos mostrou-se por volta de $10,9 \mathrm{~m} / \mathrm{s},{ }^{(78)}$ similar a média encontrada em nosso estudo. O fluxo sanguíneo muscular apresentado em nossa população, da mesma maneira, assemelha-se a valores citados na literatura em portadores de doenças cardiovasculares, como a insuficiência cardíaca $(1,64 \mathrm{~mL} / \mathrm{min} / 100 \mathrm{~mL}),{ }^{(39)}$ reforçando que a população estudada era composta por idosos com comorbidades.

Os principais indicadores associados a rigidez arterial são representados pela: 1. Pressão de pulso (PP), correspondente a diferença entre as pressões sistólica e diastólica; 2. Pressão de aumentação (PDA), refere-se à elevação absoluta de pressão de pulso devido a onda refletida; 3. Alx, representado pela diferença entre o segundo e primeiro pico de pressão na onda de pulso; ou seja, onda refletida e onda ejetora, respectivamente; 4. Alx75 e 5. VOP, que representa a velocidade em que a onda de pulso navega pelo sistema arterial e é definida pela razão da distância entre dois pontos do sistema arterial e o tempo gasto para percorrer essa distância.

A velocidade de onda de pulso é considerada o principal indicador de rigidez arterial e o índice mais utilizado pela sua melhor reprodutibilidade. ${ }^{(33)}$ Entretanto, indicadores indiretos de rigidez arterial, como PDA, Alx, Alx75 e PP central, nas últimas décadas, são de extremo valor e também associados a eventos cardiovasculares. ${ }^{(33)}$

Em nossa amostra identificamos que uma sessão aguda de exercício resistido associado a moderada restrição de fluxo foi capaz de aumentar em $13,8 \%$ o Alx e $13,0 \%$ para o Alx75, o que provavelmente foi relacionado com os maiores aumentos da PAD aórtica e PAD radial observados entres grupos. Apesar do 
ERRF ser realizado com carga de menor intensidade, há durante sua execução, estímulo metabólico mais acentuado pela restrição do fluxo sanguíneo, o que pode promover maior ativação do sistema nervoso simpático e liberação de neurotransmissores adrenérgicos, com consequente maior aumento de pressão arterial central e periférica. Ponto importante a ser citado é que também observamos em nossa amostra um aumento no delta de PP aórtica $(\Delta 7,6 \mathrm{mmHg})$, radial $(\Delta 8,2 \mathrm{mmHg}) \mathrm{e}$ pressão sistólica radial $(\Delta 6,6 \mathrm{mmHg})$ com o ERT, mas em menor valor e sem significância estatística. A intensidade que utilizamos para o treino resistido foi moderada (60\% de 1RM), como preconizado na prática diária em reabilitação cardíaca. Pode-se pressupor que cargas de maiores intensidades provoquem maiores estímulos pressóricos, similares aos ocorridos com o ERRF. Corroborando com esta hipótese, estudo recente realizado em jovens saudáveis comparando o ERRF com $30 \%$ de $1 \mathrm{RM}$ e ERT de alta intensidade a 70\% demonstrou, aumento do Alx e Alx75 em ambos os grupos. ${ }^{(80)}$ Importante ressaltar, que além da maior intensidade utilizada nesse estudo, o volume total aplicado com 4 séries e 75 repetições também foi superior ao aplicado em nosso estudo (3 séries e 45 repetições por exercício) e pode ter colaborado para um maior efeito nos indicadores indiretos da rigidez arterial em ambos os grupos.

Reforçando a hipótese de maior impacto pressórico como influenciador nos achados das variáveis Alx e Alx75 neste estudo, quando comparamos os resultados entre os grupos, o grupo ERRF demonstrou-se significativamente diferente do grupo ERT em relação a variável PAD aórtica. O aumento da pressão diastólica relaciona-se a uma maior resistência periférica, o que consequentemente pode gerar uma onda refletida mais precoce e maior Alx.

Outra possibilidade relatada na literatura e que pode interferir no aumento do Alx é referente ao tamanho do manguito utilizado para a restrição do fluxo sanguíneo. Estudo com jovens saudáveis mostrou que o ERRF imediatamente após 4 séries de exercício no banco extensor, com volume de 75 repetições e intensidade a $20 \%$ 1RM, provocou aumento significante no Alx apenas no grupo que utilizou manguitos largos $(13,5 \mathrm{~cm})$ comparado com manguitos estreitos $(5 \mathrm{~cm})$. Esses resultados não corroboram com nossos achados, pois os manguitos do método aplicado foram curtos $(5 \mathrm{~cm}) .{ }^{(81)}$ No entanto, nossa população é composta de idosos com comorbidades associadas, diferente do estudo anterior com jovens saudáveis. Importante ressaltar que não existem estudos que comparem diferentes tamanhos de manguitos em população similar à do presente estudo. Diante dos nossos resultados e 
dos apresentados em jovens, poderíamos supor que o ideal seria de fato a utilização de manguitos curtos em idosos, principalmente com comorbidades associadas, para que se evite maiores riscos durante a execução do treinamento.

A VOP não demonstrou diferenças significativas entre os momentos e entre grupos em nosso estudo. Como citado anteriormente, a VOP é um preditor de risco cardiovascular, considerado o principal indicador da rigidez arterial $^{(82)}$ e os indivíduos avaliados apresentavam variabilidade da rigidez arterial com valores similares a estudo realizado anteriormente com idosos. ${ }^{(83)}$ Esse achado associado a ausência de complicações clínicas pode sugerir segurança do protocolo de restrição de fluxo executado no presente estudo. Reforçando a hipótese em relação a importância do protocolo de exercício a ser aplicado, estudo realizado com jovens saudáveis executando handgrip a $60 \%$ da contração voluntária máxima associado a restrição de fluxo sanguíneo demonstrou aumento da VOP nessa população. ${ }^{(84)}$ Mais uma vez, diferente de nosso protocolo, o ERRF com maior intensidade aplicada (30\% de 1RM), executando os exercícios até a fadiga associada a um maior volume de treinamento (6 semanas / 3x por semana / 3-4 séries até à fadiga voluntária) em indivíduos de meia idade saudáveis, também induziu aumento da rigidez arterial periférica pela VOP. (66) Por outro lado, estudo prévio com ERRF em adultos saudáveis, mostrou redução da VOP até 30 minutos pós sessão de exercício. ${ }^{\left({ }^{85}\right)}$ Interessantemente, o protocolo de treinamento utilizou maior intensidade e volume $(40 \%$ de $1 \mathrm{RM}$ com 3 séries até à fadiga) em relação ao nosso estudo, demonstrando controvérsias na literatura e gerando outras hipóteses para essas divergências, além do protocolo de treinamento aplicado. Diferente de nossa população, nesse estudo os participantes eram jovens saudáveis.

Diante dos nossos achados em relação a indicadores da rigidez arterial e ao que existe relatado na literatura, podemos sugerir que o ERRF executado com protocolo adaptado com baixo volume e intensidade não impõe risco clínico durante sua execução, pois apesar do aumento das variáveis indiretas de Alx e Alx75, não houve modificação do indicador de maior relevância para análise da rigidez arterial, que é a VOP.

O aumento do estimulo metabólico induzido pela restrição de fluxo pode explicar uma maior ativação do sistema nervoso simpático e maior liberação de neurotransmissor adrenérgico e aumento da pressão sanguínea central e periférica, ${ }^{(45)}$ causando o aumento do Alx. 
Entretanto, estudos com restrição do fluxo sanguíneo em idosos frágeis com foco em rigidez arterial ainda são escassos na literatura e devem ser desenvolvidos para investigar se as respostas agudas observadas no presente estudo apresentarão consequências vasculares em longo prazo, principalmente na população de idosos com comorbidades cardiovasculares.

Em relação ao fluxo sanguíneo muscular e a condutância vascular no grupo ERRF também não observamos diferença significativa entre momentos e grupos, no repouso e durante a manobra de exercício isométrico - handgrip pós sessão aguda de exercício. Normalmente em repouso, o fluxo sanguíneo e a condutância vascular são 25\% menores em idosos comparados com jovens. ${ }^{\left({ }^{(8)}\right.}$ Essas diferenças significativas acontecem pelo processo que o envelhecimento desencadeia no endotélio. Normalmente, após o ERRF o sistema vascular é influenciado por aumento dos fatores angiogênicos, como: fator de crescimento endotelial vascular e fator 1 alfa induzidos pela hipóxia. ${ }^{(87)}$ Além dos fatores angiogênicos, concentrações consideráveis de NOS no sangue contribuem para o aumento da vasodilatação independente da ativação dos receptores de adenosina em indivíduos saudáveis utilizando o exercício handgrip à 10\% e $20 \%$ da CVM, durante condição de hipóxia induzida por inibidores de $\mathrm{NO}_{2}$ sintase. ${ }^{(88)} \mathrm{O}$ óxido nítrico é uma importante molécula da sinalização celular, responsável por modular o tônus vascular e envolvida no processo de angiogênese. ${ }^{(89)}$ Todas essas repostas são essenciais e influenciam diretamente a melhora do fluxo sanguíneo muscular.

O volume de treino em nosso protocolo não teve impacto no fluxo sanguíneo muscular após 1 hora da sessão. No entanto, estudo com ERRF em homens jovens saudáveis e maior volume de treino (1×30rep, 2x15rep e 1x até a fadiga $-20 \%$ de $1 \mathrm{RM}$ ), aumentou a expressão dos fatores angiogênicos: fator de crescimento endotelial vascular, fator 1 alfa induzido pela hipóxia e NOS até 4 horas pós sessão de exercício. ${ }^{(90)}$ Poderíamos supor que junto a esses resultados pudesse ocorrer aumento do fluxo sanguíneo muscular, entretanto essa variável não foi mensurada. Por outro lado, não podemos deixar de citar o fato de serem populações extremamente opostas, jovens saudáveis versus idosos com comorbidades. Como citado anteriormente, o processo de envelhecimento promove diversas modificações no sistema endotelial, o que pode ser responsável por uma não resposta ou menor resposta a esse tipo de treinamento, independente do volume e intensidade de treinamento utilizados. Apesar do fluxo sanguíneo ser uma variável fundamental na análise da segurança vascular em 
idosos frágeis, estudos agudos não foram realizados ainda nessa população, não sendo possível uma análise crítica desses achados.

Entretanto, cronicamente, estudos mostram que o ERRF até a fadiga comparado com ERT de baixa intensidade (20\% 1RM) pode resultar na melhora do fluxo sanguíneo muscular em mulheres jovens e idosos fisicamente ativos. ${ }^{(69,91)}$ Chegar à fadiga máxima em cada série, aumenta o volume de repetições e consequentemente maior acúmulo de metabólitos. Outro ponto que merece discussão em relação ao treinamento é o tipo de exercício executado. O exercício aeróbico associado a restrição do fluxo sanguíneo em homens fisicamente ativos, induziu maior disponibilidade de óxido nítrico no sangue e melhora da função antioxidante. ${ }^{(92)}$ Portanto, o tipo de exercício também pode influenciar na melhora do fluxo sanguíneo muscular, pela maior produção plasmática de substâncias vasodilatadoras. Vale ressaltar que os indivíduos desses estudos eram fisicamente ativos e sabidamente um dos mais importantes efeitos do treinamento físico, principalmente do aeróbico, é a melhora da função endotelial.

A intensidade de treinamento, apesar de ser uma variável que interfere na alteração do fluxo sanguíneo muscular não mostrou diferença estatisticamente significante no grupo ERT em nosso estudo. Estudos na literatura com ERT em alta intensidade (70\% 1RM) quando comparado com o ERRF e ERT com baixa intensidade (20\% 1RM), demonstrou o aumento do fluxo sanguíneo muscular em homens jovens ativos sem afetar a complacência arterial sistêmica. ${ }^{\left({ }^{93}\right)}$ Além disso, o ERT com intensidade ( $80 \%$ de 1RM) em mulheres hipertensas apresentou maior fluxo sanguíneo muscular comparado com ERT de moderada intensidade (50\% de 1RM). ${ }^{(41)}$ Nesse estudo, a melhora do fluxo sanguíneo muscular veio acompanhada com o aumento da FC pós exercício. Nosso protocolo, por utilizar menor intensidade no exercício $(60 \%$ de $1 \mathrm{RM})$, provavelmente não estimulou metabólicos e fatores miogênicos suficientes para melhorar o fluxo sanguíneo muscular. Sendo assim, cargas $\geq 70 \%$ de 1 RM aplicadas na sessão de exercício, parecem melhorar o fluxo sanguíneo muscular nessas populações, estimulando mais metabólitos como: lactado e íons de hidrogênio e consequentemente ativando fatores miogênicos e produzindo mais NOS.

Diante do que foi exposto, volume de treino, intensidade e tipo de exercício, podem intensificar a melhora da vasodilatação muscular em relação ao ERRF e ERT, mas talvez essa ação se dê principalmente ao longo do tempo, como um 
efeito de treinamento e não após uma única sessão. No contexto do fluxo sanguíneo muscular podemos considerar o ERRF seguro, com ação neutra sob essa variável.

Quanto as variáveis hemodinâmicas não evidenciamos diferença significativa no grupo ERRF. Entretanto, a FC aumentou em todos os momentos pós sessão de exercício no grupo ERT. Esse aumento da FC pós sessão no grupo ERT pode ter sido ocasionado pela intensidade usada do exercício. Habitualmente, durante o ERT ocorre elevação progressiva da FC. ${ }^{(94)}$ O ERT aplicado em 2 intensidades diferentes (50\% - 80\% de 1RM) em mulheres hipertensas, mostrou que ambos os grupos aumentam a FC pós exercício. No entanto, o ERT de alta intensidade evidenciou aumento significativo em relação ao ERT com intensidade moderada. ${ }^{(41)}$ Nossos participantes eram idosos frágeis sedentários, sendo assim a intensidade que utilizamos $(60 \%$ de $1 \mathrm{RM})$, pode ter proporcionado uma sobrecarga cardíaca significativa nos momentos pós sessão de exercício. No entanto, os valores da pressão arterial não foram alterados entre grupos e momentos.

Importante ressaltar que a PAD no momento 60 minutos foi estatisticamente significativa apenas no grupo ERRF. A importância clínica desse achado parece inexpressiva, pois a variação ocorrida na pressão diastólica foi dentro de valores considerados normais, sendo difícil supor risco ao grupo que executou o ERRF. Enquanto houve um aumento no delta da PAD $(\Delta 1,2 \mathrm{mmHg})$ no grupo ERRF, o grupo ERT apresentou uma queda dessa variável $(\Delta-1,7 \mathrm{mmHg})$, o que pode ter sido responsável pela diferença significativa entre os grupos.

Diferente dos nossos resultados, o aumento de todos os parâmetros hemodinâmicos ocorreu durante o ERRF (20\% 1RM) em adultos saudáveis em relação ao ERT de baixa intensidade $(20 \% 1 \mathrm{RM}) .{ }^{(87)}$ Nesse estudo, os valores de lactato e noradrenalina plasmática foram elevados até 30 minutos apenas no grupo ERRF, possivelmente pelo protocolo de treino utilizado $(1 \times 30$ rep $+3 x$ até à fadiga $-20 \%$ de 1RM). Habitualmente, durante o ERRF valores maiores de PAS e PAD são demonstrados em relação ao ERT com intensidade $\geq 60 \%$ de $1 \mathrm{RM}$, principalmente em pacientes com hipertensão arterial sistêmica. ${ }^{(95)}$ Além disso, durante o ERRF com baixa intensidade $(20 \% 1 \mathrm{RM})$ comparado com alta e baixa intensidade sem restrição do fluxo sanguíneo (70\%-20\% 1RM), ocorreu aumento significativo na PAS, PAD e PAM em mulheres idosas saudáveis. Curiosamente, o exercício leg press causou maior stress hemodinâmico, principalmente na variável PAD em comparação com o exercício banco extensor, indicando que pode ocorrer alterações hemodinâmicas em diferentes 
exercícios. ${ }^{(96)}$ Outro estudo demonstra que durante o ERRF com baixa intensidade (20\% 1RM) há maior stress hemodinâmico em idosos e jovens sedentários comparado com a caminhada associada a restrição parcial do fluxo sanguíneo, indicando que o exercício aeróbico combinado com a técnica de restrição do fluxo sanguíneo pode promover menor stress hemodinâmico para a população idosa. ${ }^{(97)}$

O protocolo de sessão de exercício resistido utilizado no presente estudo foi adaptado para nossa amostra e o volume de séries e repetições é inferior ao volume de treino dos estudos citados. Enquanto a maioria dos estudos realizam 4 séries com um total de 60-75 repetições por exercício, o nosso protocolo consiste em 3 séries com um total de 45 repetições. Essa seria uma possível explicação para não ocorrer variações dos parâmetros hemodinâmicos após o ERRF em nosso estudo. Agudamente o ERRF não demonstrou mudanças significativas nas variáveis hemodinâmicas, podendo ser uma metodologia segura para idosos frágeis, sem produzir stress hemodinâmico e eventos adversos cardiovasculares.

Nosso estudo possui algumas limitações que devem ser reportadas. O tamanho da amostra foi de conveniência e pequeno para demonstrar maior poder dos dados. Outro ponto importante foi relacionado ao protocolo de treinamento. Como o protocolo utilizado foi adaptado para nossa amostra, com menor volume de treino quando comparado a outros estudos, e protocolos de ERRF em idosos frágeis ainda são inexistentes é possível supor que algumas variáveis não tiveram alterações significativas, principalmente no fluxo sanguíneo muscular e parâmetros hemodinâmicos pós exercício por este motivo. Por outro lado, o protocolo escolhido foi baseado em trabalho com população que mais se aproximava da que estudamos, portadores de síndrome metabólica, visando a segurança da aplicação desse tipo de exercício em população tão especial, idosos com comorbidades. Podemos considerar que a escolha foi correta, uma vez que tal protocolo não gerou prejuízo na maior parte das variáveis estudadas. Entretanto, será fundamental verificar se tal protocolo será eficaz em relação aos efeitos de treinamento, principalmente em desfechos clínicos como força muscular e velocidade de marcha desses idosos. 


\section{CONCLUSÕES}

1. Uma sessão aguda de exercício resistido com moderada restrição do fluxo sanguíneo promoveu aumento significativo nos indicadores indiretos de rigidez arterial pressão de aumentação, pressão diastólica radial, pressão diastólica aórtica, Alx e Alx75. No entanto, não provocou impacto na velocidade da onda de pulso em relação ao exercício resistido tradicional em idosos com baixa velocidade de marcha;

2. Uma sessão aguda de exercício resistido com moderada restrição do fluxo sanguíneo não promoveu alteração significativa no fluxo sanguíneo muscular e condutância vascular em relação ao exercício resistido tradicional em idosos com baixa velocidade de marcha;

3. O exercício resistido tradicional promoveu aumento significativo da frequência cardíaca até uma hora após a sessão do exercício, mas não houve diferença significativa em relação ao exercício resistido com moderada restrição do fluxo sanguíneo. 


\section{REFERÊNCIAS}

1. Ganguly S. Good health adds life to years. J Indian Med Assoc. 2012;110(4):212-3.

2. Kovacic JC, Moreno P, Nabel EG, Hachinski V, Fuster V, Park DC, et al. Cellular senescence, vascular disease, and aging part 2 of a 2-part review: clinical vascular disease in the elderly. Circulation. 2011;1(17):1900-10.

3. Park DC, Yeo SG. Aging. Korean J Audiol. 2013;17(2):39-44.

4. Cederholm T. Overlaps between frailty and sarcopenia definitions. Nestle Nutr Inst Workshop Ser. 2015;83:65-9.

5. Keevil VL, Romero-Ortuno R. Ageing well: a review of sarcopenia and frailty. Proc Nutr Soc. 2015;74(4):337-47.

6. Studenski S, Perera S, Patel K, Rosano C, Faulkner K, Inzitari M, et al. Gait speed and survival in older adults. JAMA. 2015;305(1):50-8.

7. Lee L, Heckman G, Molnar FJ. Frailty. 2015;61:227-31.

8. Fielding RA, Rejeski WJ, Blair S, Church T, Espeland MA, Gill TM, et al.; LIFE Research Group. The lifestyle interventions and independence for elders study: design and methods. J Gerontol A Biol Sci Med Sci. 2011;66(11):1226-37.

9. Fragala MS, Cadore EL, Dorgo S, Izquierdo M, Kraemer WJ, Peterson MD, et al. Resistance training for older adults: position statement from the National Strength and Conditioning Association. J Strength Cond Res. 2019;33(8):2019-52.

10. Cortez-Cooper MY, Anton MM, Devan AE, Neidre DB, Cook JN, Tanaka H. The effects of strength training on central arterial compliance in middle-aged and older adults. Eur $\mathrm{J}$ Cardiovasc Prev Rehabil. 2008;15(2):149-55.

11. Centner C, Wiegel P, Gollhofer A, Konig D. Effects of blood flow restriction training on muscular strength and hypertrophy in older individuals: a systematic review and meta-analysis. Sport Med. 2019;49(1):95-108.

12. Amorim S, Gaspar AP, Degens H, De Matos LD. The effects of blood flow restriction exercise on vascular function in the elderly: a systematic review. Clin Integr Med. 2019;3:1-6.

13. Fried LP, Carlson MC, Freedman M, Frick KD, Glass TA, Hill J, et al. A social model for health promotion for an aging population: initial evidence on the Experience Corps model. $\mathrm{J}$ Urban Health. 2004;81(1):64-78.

14. Brasil. Ministério da Saúde. Atenção à saúde da pessoa idosa e envelhecimento. Brasília (DF): Ministério da Saúde; 2010.

15. Martinez-Ramirez A, Martinikorena I, Gomez M, Lecumberri P, Millor N, Rodriguez-Manas $\mathrm{L}$, et al. Frailty assessment based on trunk kinematic parameters during walking. J Neuroeng Rehabil.. 2015;12:48.

16. Ricci NA. Frailty and cardiovascular risk in community- dwelling elderly : a population-based study. 2014;4(9):1677-85.

17. Wu I, Lin C, Hsiung CA. Emerging roles of frailty and inflammaging in risk assessment of age-related chronic diseases in older adults: the intersection between aging biology and personalized medicine. Biomedicine (Taipei). 2015;5(1):1-10. 
18. Cadore EL, Rodriguez-Manas L, Sinclair A, Izquierdo M. Effects of different exercise interventions on risk of falls, gait ability, and balance in physically frail older adults: a systematic review. Rejuvenation Res. 2013;16(2):105-14.

19. Chen X, Mao G, Leng SX. Frailty syndrome: an overview. Clin Interv Aging. 2014;9(9):43341.

20. Nazari-Shafti TZ, Cooke JP. Telomerase therapy to reverse cardiovascular senescence. 2015;(3):172-5.

21. Miljkovic N, Lim J. Aging of skeletal muscle fibers. 2015;39(2):155-62.

22. Odden MC, Peralta CA, Haan MN, Covinsky KE. Rethinking the association of high blood pressure with mortality in elderly adults: the impact of frailty. Arch Intern Med.

2012;172(15):1162-8.

23. Manini TM, Visser M, Won-Park S, Patel KV, Strotmeyer ES, Chen H, et al. Knee extension strength cutpoints for maintaining mobility. 2007;451-7.

24. Lexell J. Human aging, muscle mass, and fiber type composition. J Gerontol A Biol Sci Med Sci. 1995;50 Spec No:11-6.

25. Verdijk LB, Koopman R, Schaart G, Meijer K, Savelberg HH, van Loon LJ. Satellite cell content is specifically reduced in type II skeletal muscle fibers in the elderly. Am J Physiol Endocrinol Metab. 2007;292(1):E151-7.

26. Frontera WR, Suh D, Krivickas LS, Hughes VA, Goldstein R, Roubenoff R, et al. Skeletal muscle fiber quality in older men and women. 2000;279(3):611-8.

27. D'Antona G, Pellegrino MA, Adami R, Rossi R, Carlizzi CN, Canepari M, et al. The effect of ageing and immobilization on structure and function of human skeletal muscle fibres. J Physiol. 2003;552(Pt 2):499-511.

28. Tesauro M, Mauriello A, Rovella V, Cardillo C, Melino G, Daniele N Di. Arterial ageing: from endothelial dysfunction to vascular calcification. J Intern Med. 2017;281(5):471-82.

29. Nakayama H, Nishida K, Otsu K. Macromolecular degradation systems and cardiovascular aging. Circ Res. 2016;118(10):1577-92.

30. Mitchell GF, Parise H, Benjamin EJ, Larson MG, Keyes MJ, Vita JA, et al. Changes in arterial stiffness and wave reflection with advancing age in healthy men and women the framingham heart study. Hypertension. 2004;43(6):1239-45.

31. Jia G, Aroor AR, Jia C, Sowers JR. Endothelial cell senescence in aging-related vascular dysfunction. Biochim Biophys Acta Mol Basis Dis. 2019;1865(7):1802-9.

32. Vlachopoulos C, Aznaouridis K, Stefanadis C. Prediction of cardiovascular events and allcause mortality with arterial stiffness: a systematic review and meta-analysis. J Am Coll Cardiol. 2010;55(13):1318-27.

33. Mendes-Pinto D, Rodrigues-Machado MG. Applications of arterial stiffness markers in peripheral arterial disease. J Vasc Bras. 2019;18:e20180093.

34. Pierce DR, Doma K, Leicht AS. Acute effects of exercise mode on arterial stiffness and wave reflection in healthy young adults: a systematic review and meta-analysis. Front Physiol. 2018;9:73. 
35. Figueroa A, Okamoto T, Jaime SJ, Fahs CA. Impact of high- and low-intensity resistance training on arterial stiffness and blood pressure in adults across the lifespan: a review. Eur $\mathrm{J}$ Physiol. Pflugers Arch. 2019;471(3):467-78.

36. Gardner AW, Katzel LI, Sorkin JD, Bradham DD, Hochberg MC, Flinn WR, et al. Exercise rehabilitation improves functional outcomes and peripheral circulation in patients with intermittent claudication: a randomized controlled trial. J Am Geriatr Soc. 2001;49(6):755-62.

37. Joyner MJ, Dietz NM, Shepherd JT,. From belfast to Mayo and beyond: the use and future of plethysmography to study blood flow in human limbs. J Appl Physiol (1985). 2019;(13):243141.

38. Salisbury DL, Brown RJ, Bronas UG, Kirk LN, Treat-Jacobson D. Measurement of peripheral blood flow in patients with peripheral artery disease: methods and considerations. Vasc Med. 2018;23(2):163-71.

39. Antunes-Correa LM, Kanamura BY, Melo RC, Nobre TS, Ueno LM, Franco FG, et al. Exercise training improves neurovascular control and functional capacity in heart failure patients regardless of age. Eur J Prev Cardiol. 2012;19(4):822-9.

40. O'Leary DS. Regional vascular resistance vs. conductance: which index for baroreflex responses? Am J Physiol. 1991;260(2 Pt 2):H632-7.

41. Brito AF, Brasileiro-Santos MS, Oliveira CV, Nobrega TK, Forjaz CL, Santos AC. Highintensity resistance exercise promotes postexercise hypotension greater than moderate intensity and affects cardiac autonomic responses in women who are hypertensive. J Strength Cond Res. 2015;29(12):3486-93.

42. Fahs CA, Rossow LM, Loenneke JP, Thiebaud RS, Kim D, Bemben DA, et al. Effect of different types of lower body resistance training on arterial compliance and calf blood flow. Clin Physiol Funct Imaging. 2012;32(1):45-51.

43. Romero SA, Minson CT, Halliwill JR. The cardiovascular system after exercise. J Appl Physiol (1985). 2017;122(4):925-32.

44. Thiebaud RS, Fahs CA, Rossow LM, Loenneke JP, Kim D, Mouser JG, et al. Effects of age on arterial stiffness and central blood pressure after an acute bout of resistance exercise. Eur $\mathrm{J}$ Appl Physiol. 2016;116(1):39-48.

45. Spranger MD, Krishnan AC, Levy PD, OLeary DS, Smith SA. Blood flow restriction training and the exercise pressor reflex: a call for concern. Am J Physiol Heart Circ Physiol. 2015;309(9):H1440-52.

46. Haskell WL, Lee IM, Pate RR, Powell KE, Blair SN, Franklin BA, et al. Physical activity and public health: updated recommendation for adults from the American College of Sports Medicine and the American Heart Association. Circulation. 2007;116(9):1081-93.

47. Miyachi M, Kawano H, Sugawara J, Takahashi K, Hayashi K, Yamazaki K, et al. Unfavorable effects of resistance training on central arterial compliance: a randomized intervention study. Circulation. 2004;110(18):2858-63.

48. Collier SR, Kanaley JA, Carhart R Jr, Frechette V, Tobin MM, Hall AK, et al. Effect of 4 weeks of aerobic or resistance exercise training on arterial stiffness, blood flow and blood pressure in pre- and stage-1 hypertensives. J Hum Hypertens. 2008;22(10):678-86.

49. Cortez-Cooper MY, DeVan AE, Anton MM, Farrar RP, Beckwith KA, Todd JS, et al. Effects of high intensity resistance training on arterial stiffness and wave reflection in women. Am J Hypertens. 2005;18(7):930-4. 
50. Wernbom M. Effects of an acute bout of low-load resistance training with blood flow restriction: -with special reference to muscle damage, hypertrophic signaling and satellite cells [dissertation]. [Oslo]: Norwegian School of Sports Sciences; 2011. $115 \mathrm{f}$.

51. Nakajima T, Kurano M, lida H, Takano H, Madarame H, Yasuda T, et al. Effects of lowintensity KAATSU resistance training on skeletal muscle size and muscle strength/endurance capacity in patients with ischemic heart diseases. Med Sci Sports Exerc. 2010;42(5 Suppl 1):743.

52. Scott BR, Loenneke JP, Slattery KM, Dascombe BJ. Blood flow restricted exercise for athletes: a review of available evidence. J Sci Med Sport. 2016;19(5):360-7.

53. Fry CS, Glynn EL, Drummond MJ, Timmerman KL, Fujita S, Abe T, et al. Blood flow restriction exercise stimulates mTORC1 signaling and muscle protein synthesis in older men. $J$ Appl Physiol (1985). 2010;108(5):1199-209.

54. Karabulut M, Sherk VD, Bemben DA, Bemben MG. Inflammation marker, damage marker and anabolic hormone responses to resistance training with vascular restriction in older males. Clin Physiol Funct Imaging. 2013;33(5):393-9.

55. Mattar MA, Gualano B, Perandini LA, Shinjo SK, Lima FR, Sa-Pinto AL, et al. Safety and possible effects of low-intensity resistance training associated with partial blood flow restriction in polymyositis and dermatomyositis. Arthritis Res Ther. 2014;16(5):473.

56. Satoh I. Kaatsu Training: application to metabolic syndrome. Int J KAATSU Train Res. $2011 ; 7(1): 7-12$.

57. Fukuda T, Yasuda T, Fukumura K, lida H, Morita T, Sato Y, et al. Low-intensity kaatsu resistance exercises using an elastic band enhance muscle activation in patients with cardiovascular diseases. Int J KAATSU Train Res. 2013;9(1):1-5.

58. Buford TW , Fillingim RB, Manini TM, Sibille KT, Vincent KR, Wu SS. Kaatsu training to enhance physical function of older adults with knee osteoarthritis: design of a randomized controlled trial. Contemp Clin Trials. 2015;43:217-22.

59. Segal NA, Williams GN, Davis MC, Wallace RB, Mikesky AE. Efficacy of blood flowrestricted, low-load resistance training in women with risk factors for symptomatic knee osteoarthritis. PM R. 2015;7(4):376-84.

60. Libardi CA, Chacon-Mikahil MP, Cavaglieri CR, Tricoli V, Roschel H, Vechin FC, et al. Effect of concurrent training with blood flow restriction in the elderly. Int J Sports Med.

2015;36(5):395-9.

61. Takarada Y, Takazawa H, Sato Y, Takebayashi S, Tanaka Y, Ishii N. Effects of resistance exercise combined with moderate vascular occlusion on muscular function in humans. J Appl Physiol (1985). 2000;88(6):2097-106.

62. Ozaki H, Loenneke JP, Thiebaud RS, Stager JM, Abe T. Possibility of leg muscle hypertrophy by ambulation in older adults: a brief review. Clin Interv Aging. 2013;8:369-75.

63. Yokokawa $\mathrm{Y}$, Hongo M, Urayama H, Nishimura T, Kai I. Effects of low-intensity resistance exercise with vascular occlusion on physical function in healthy elderly people. Biosci Trends. 2008;2(3):117-23.

64. Horiuchi M, Okita K. Blood flow restricted exercise and vascular function. Int J Vasc Med. 2012;2012:543218. 
65. Park SY, Kwak YS, Harveson A, Weavil JC, Seo KE. Low intensity resistance exercise training with blood flow restriction: insight into cardiovascular function, and skeletal muscle hypertrophy in humans. Korean J Physiol Pharmacol. 2015;19(3):191-6.

66. Fahs CA, Rossow LM, Thiebaud RS, Loenneke JP, Kim D, Abe T, et al. Vascular adaptations to low-load resistance training with and without blood flow restriction. Eur J Appl Physiol. 2014;114(4):715-24.

67. Yasuda T, Fukumura K, Tomaru T, Nakajima T. Thigh muscle size and vascular function after blood flow-restricted elastic band training in older women. Oncotarget. 2016;7(23):33595607.

68. Shimizu R, Hotta K, Yamamoto S, Matsumoto T, Kamiya K, Kato M, et al. Low-intensity resistance training with blood flow restriction improves vascular endothelial function and peripheral blood circulation in healthy elderly people. Eur J Appl Physiol. 2016;116(4):749-57.

69. Patterson SD, Ferguson RA. Enhancing strength and postocclusive calf blood flow in older people with training with blood-flow restriction. J Aging Phys Act. 2011;19(3):201-13.

70. Kim J, Lang JA, Pilania N, Franke WD. Effects of blood flow restricted exercise training on muscular strength and blood flow in older adults. Exp Gerontol. 2017;99:127-32.

71. Choi KM. Sarcopenia and sarcopenic obesity. Korean J Intern Med. 2016;31(6):86-9.

72. Nakajima T, Morita T, Sato Y. Key considerations when conducting KAATSU training. Int J KAATSU Train Res. 2011;7(1):1-6.

73. Radaelli R, Botton CE, Wilhelm EN, Bottaro M, Brown LE, Lacerda F, et al. Time course of low- and high-volume strength training on neuromuscular adaptations and muscle quality in older women. Age (Dordr). 2014;36(2):881-92.

74. Stewart A, Marfell-Jones M, Olds T, De Ridder H; International Society for Advancement of Kinanthropometry. International standards for anthropometric assessment. Lower Hutt: International Society for the Advancement of Kinanthropometry; 2011.

75. KAATSU user manual: including KAATSU protocols for training, recovery, rehabilitation, fitness, and wellness. Tokyo: KAATSU Global; 2017.

76. Wythe S, Davies T, Martin D, Feelisch M, Gilbert-Kawai E. Getting the most from venous occlusion plethysmography: proposed methods for the analysis of data with a rest/exercise protocol. Extrem Physiol Med. 2015;4:8.

77. D. E. Hokanson. Al6: arterial inflow system manual. Bellevue: D. E. Hokanson; c2014.

78. David M, Malti O, AIGhatrif M, Wright J, Canepa M, Strait JB. Pulse wave velocity testing in the Baltimore longitudinal study of aging. J Vis Exp. 2014;(84): e50817. Erratum in: $J$ Vis Exp. 2014;(87):e53421.

79. AtCor Medical. Research applications manual. Sydney: AtCor Medical; c2010.

80. Tai YL, Marshall EM, Glasgow A, Parks JC, Sensibello L, Kingsley JD. Pulse wave reflection responses to bench press with and without practical blood flow restriction. Appl Physiol Nutr Metab. 2019;44(4):341-7.

81. Rossow LM, Fahs CA, Loenneke JP, Thiebaud RS, Sherk VD, Abe T, et al. Cardiovascular and perceptual responses to blood-flow-restricted resistance exercise with differing restrictive cuffs. Clin Physiol Funct Imaging. 2012;32(5):331-7. 
82. Townsend RR, Wilkinson IB, Schiffrin EL, Avolio AP, Chirinos JA, Cockcroft JR, et al. Recommendations for improving and standardizing vascular research on arterial stiffness: a scientific statement from the American Heart Association. Hypertension. 2015;66(3):698-722.

83. Perissiou M, Bailey TG, Windsor M, Leicht AS, Golledge J, Askew CD. Reliability of arterial stiffness indices at rest and following a single bout of moderate-intensity exercise in older adults. Clin Physiol Funct Imaging. 2019;39(1):42-50.

84. Credeur DP, Jones R, Stanford D, Stoner L, McCoy S, Jessee M. Central cardiovascular hemodynamic response to unilateral handgrip exercise with blood flow restriction. Eur J Appl Physiol. 2019;119(10):2255-63.

85. Figueroa A, Vicil F. Post-exercise aortic hemodynamic responses to low-intensity resistance exercise with and without vascular occlusion. Scand J Med Sci Sports. 2011;21(3):431-6.

86. Dinenno FA, Jones PP, Seals DR, Tanaka H. Limb blood flow and vascular conductance are reduced with age in healthy humans declines in oxygen demand. Circulation. 1999;100(2):164-70.

87. Takano H, Morita T, lida H, Asada K, Kato M, Uno K, et al. Hemodynamic and hormonal responses to a short-term low-intensity resistance exercise with the reduction of muscle blood flow. Eur J Appl Physiol. 2005;95(1):65-73.

88. Casey DP, Madery BD, Curry TB, Eisenach JH, Wilkins BW, Joyner MJ. Nitric oxide contributes to the augmented vasodilatation during hypoxic exercise. J Physiol. 2010;588(Pt 2):373-85.

89. Segal SS. Regulation of blood flow in the microcirculation. Microcirculation. 2005;12(1):3345.

90. Ferguson RA, Hunt JE, Lewis MP, Martin NR, Player DJ, Stangier C, et al. The acute angiogenic signalling response to low-load resistance exercise with blood flow restriction. Eur $\mathrm{J}$ Sport Sci. 2018;18(3):397-406.

91. Patterson SD, Ferguson RA. Increase in calf post-occlusive blood flow and strength following short-term resistance exercise training with blood flow restriction in young women. Eur J Appl Physiol. 2010;108(5):1025-33.

92. Christiansen D, Eibye KH, Hostrup M, Bangsbo J. Blood flow-restricted training enhances thigh glucose uptake during exercise and muscle antioxidant function in humans. Metabolism. 2019;98:1-15.

93. Fahs CA, Rossow LM, Seo DI, Loenneke JP, Sherk VD, Kim E, et al. Effect of different types of resistance exercise on arterial compliance and calf blood flow. Eur J Appl Physiol. 2011;111(12):2969-75.

94. MacDougall JD, Sutton JR. Arterial blood pressure response to heavy resistance exercise. J Appl Physiol (1985). 1985;58(3):785-90.

95. Domingos E, Polito MD. Blood pressure response between resistance exercise with and without blood flow restriction: a systematic review and meta-analysis. Life Sci. 2018;209:12231.

96. Scott BR, Peiffer JJ, Thomas HJ, Marston KJ, Hill KD, Snow RJ, et al. Hemodynamic responses to low-load blood flow restriction and unrestricted high-load resistance exercise in older women. 2018;9:1324.. 
97. Staunton CA, May AK, Brandner CR, Warmington SA. Haemodynamics of aerobic and resistance blood flow restriction exercise in young and older adults. Eur J Appl Physiol. 2015;115(11):2293-302. 


\begin{abstract}
Introduction: Acute high-intensity resistance training causes changes in vascular system and hemodynamic behaviour, which are not interesting for older people and those with comorbidities. New training strategies have appeared by combining low intensity exercises with moderate blood flow restriction, such as the KAATSU. However, the effects of resistance exercises on arterial stiffness and hemodynamic behaviour in elderly people with comorbidities and reduced physical performance remain unknown. Purpose: To determine the effects of acute resistance exercise with moderate blood flow restriction on central blood pressure, arterial stiffness and muscle blood flow in elderly people with low gait speed, and to verify the blood pressure and heart rate up to 1 hour after the exercise session. Methods: This was a randomized controlled trial including two groups, the traditional resistance exercise $(60 \%$ of $1 \mathrm{RM})$ and the low intensity resistance exercise training with blood flow restriction ( $20 \%$ of $1 \mathrm{RM})$. Before and after 1 hour exercise session, participants' central blood pressure, arterial stiffness, muscle blood flow, blood pressure, and hear rate were measured. The groups were compared for initial measurements using the Fisher's exact test for qualitative variables and the Student's $t$ test for quantitative variables. For the measures observed more than once, we used generalized estimating equation models. A $p$ value $<0.05$ was considered statistically significant, and means and standard deviations data were presented. Results: The sample included 17 older individuals (3 men and 14 women). Of these, 7 were in the TRE group and 10 in the LIRE-BFR group.The resistance exercise with moderate blood flow restriction in a comparison with the resistance traditional exercise showed aortic augmentation index $(p=0.008)$, aortic augmentation index corrected for heart rate 75 (0.014), and diastolic pressure $(p=0.029)$, and radial blood pressure $(p=0.017)$. However, no significant changes occurred between groups regarding pulse wave velocity, muscle blood flow and hemodynamic variables evaluated up to 1 hour after the exercise session. Conclusion: An acute session of resistance exercise with moderate blood flow restriction promotes increase in indirect index of arterial stiffness, aortic augmentation index, and aortic augmentation index corrected for heart rate 75 . However, the session did not cause changes in pulse wave velocity, muscle blood flow and hemodynamic variables among elderly people with low gait speed.
\end{abstract}


Clinical Trials Identifier: NCT03272737

Keywords: KAATSU (not in DeCS); Vascular stiffness; Regional blood flow; Gait; Aged 